Distributional and local limit laws for a class of iterated maps that contract on average

Santos, Sara I and Walkden, Charles

2011

MIMS EPrint: 2011.39

Manchester Institute for Mathematical Sciences

School of Mathematics

The University of Manchester

\footnotetext{
Reports available from: http://eprints.maths.manchester.ac.uk/

And by contacting: The MIMS Secretary

School of Mathematics

The University of Manchester

Manchester, M13 9PL, UK
} 


\title{
Distributional and local limit laws for a class of iterated maps that contract on average
}

\author{
Sara I. Santos*† and Charles Walkden*
}

26th March 2011

\begin{abstract}
We consider iterated function schemes that contract on average with place-dependent probabilities. We are interested in generalisations of the central limit theorem, particularly to observations with infinite variance. By studying the spectral properties of an associated one-parameter family of transfer operators acting on an appropriate function space, we prove both a distributional and local limit law with convergence to a stable distribution.
\end{abstract}

\section{$\S 1 \quad$ Introduction}

The study of the limiting behaviour of the sum of a sequence of observations of random variables is a key problem in dynamical systems and probability theory. For example, the ergodic theorem describes the average behaviour of such sums. In the case where the observation has finite variance, the central limit theorem then describes the how these sums are distributed around their expected value, namely convergence in distribution to a normal distribution. More generally, if the observation does not have a finite variance, then one can ask about convergence in distribution to a stable law. Stable limit laws have been well-understood for i.i.d. random variables [IL, for example], however there has been much recent interest in analogues of such results in dynamical systems, particularly in hyperbolic and non-uniformly hyperbolic systems [AD2, Gou, for example] and for random walks on the affine group of the real line [GP]. In this note we study iterated function schemes (IFS) with place-dependent probabilities that satisfy a 'contractionon-average' condition. Central limit theorems, and generalisations thereof,

\footnotetext{
*School of Mathematics, The University of Manchester, Oxford Road, Manchester, M13 9PL, U.K.

${ }^{\dagger}$ An earlier version of the results in this paper appeared in the first author's $\mathrm{PhD}$ thesis. Financially supported by Fundação para a Ciência e Tecnologia, Portugal, grant SFRH/BD/6921/2001.
} 
for IFSs that contract-on-average have been studied in [Pe, HH2, W]. Such systems are of interest in a wide range of situations, see $[\mathrm{DF}]$ and the references cited therein. We discuss stable limit laws in the context of IFSs that contract on average. We also prove a local version of the stable limit law. The methodology uses the spectral properties of a one-parameter family of transfer operators $P_{t}$. By studying $P_{t}$ on an appropriate function space, motivated by [HH1, HH2, GP], we prove that $P_{t}$ is a quasi-compact operator with a simple maximal eigenvalue $\lambda_{t}$. We then apply a result from [AD1, AD2] to study the asymptotic expansion of $\lambda_{t}$, allowing us to relate $\lambda_{t}$ to the sums in question.

\section{$\S 2 \quad$ Statement of results}

Let $(X, d)$ be a locally compact (but not necessarily compact) second countable metric space. Consider a finite or countable family of Lipschitz maps $T_{j}: X \rightarrow X, 1 \leq j<M$ (where $M \leq \infty$ ). We are interested in studying the statistical properties of the iterated function scheme (IFS) formed by applying the maps $T_{j}$ chosen at random according to place-dependent Markov transition probabilities.

Let $p_{j}: X \rightarrow[0,1]$ be continuous maps such that $\sum_{j} p_{j}(x)=1$ for each $x \in X$. Define a Markov transition probability by

$$
p(x, A)=\sum_{j} p_{j}(x) \chi_{A}\left(T_{j} x\right)
$$

for each Borel subset $A \subset X$. (Here $\chi_{A}$ denotes the characteristic function of $A$.)

We say that the system contracts on average after 1 step if there exists $r \in(0,1)$ such that

$$
\sup _{x, y, z \in X, y \neq z} \sum_{j} p_{j}(x) d\left(T_{j} y, T_{j} z\right) \leq r d(y, z) .
$$

Remark. More precisely, we could refer to (1) as arithmetic contraction on average. It is strictly stronger than a logarithmic contraction on average condition, namely

$$
\sup _{x, y, z \in X, y \neq z} \sum_{j} p_{j}(x) \log d\left(T_{j} y, T_{j} z\right) \leq r \log d(y, z),
$$

as assumed in [BDEG].

More generally, we will consider IFSs that contract on average after $n_{0}$ steps; see $\S 3.1$.

We also assume that the $p_{j} \geq 0$ are continuous and satisfy a Dini condition (cf. [E1]). 
With these assumptions, together with the mild technical assumptions in $\S 3.1$, it is known [BDEG, Pe] that there exists a unique attractive stationary Borel probability measure $\nu$ on $X$, i.e. for all Borel sets $A$

$$
\int p(x, A) d \nu(x)=\nu(A) .
$$

Moreover, for any $x_{0} \in X$, we have that $\int d\left(x, x_{0}\right) d \nu(x)<\infty$. Let

$$
\theta_{0}=\sup \left\{\theta>0 \mid \int d\left(x, x_{0}\right)^{\theta} d \nu(x)<\infty \text { for all } x_{0} \in X\right\} \text {. }
$$

Note that $\theta_{0}>1$ and that $\int d\left(x, x_{0}\right)^{\theta} d \nu(x)<\infty$ for all $\theta<\theta_{0}$.

Let $\Sigma=\left\{\mathbf{j}=\left(j_{1}, j_{2}, \ldots\right) \mid 1 \leq j_{k}<M\right\}$ denote the one-sided full-shift. Define a cylinder set by $\left[j_{1}, j_{2}, \ldots, j_{n}\right]=\left\{\mathbf{i}=\left(i_{k}\right) \in \Sigma \mid i_{k}=j_{k}, 1 \leq k \leq n\right\}$. For each $x \in X$ we define a probability measure $\mu_{x}$ on $\Sigma$ by defining $\mu_{x}$ on cylinder sets by

$$
\mu_{x}\left[j_{1}, j_{2}, \ldots, j_{n}\right]=p_{j_{1}}(x) p_{j_{2}}\left(T_{j_{1}} x\right) \cdots p_{j_{n}}\left(T_{j_{n-1}} \cdots T_{j_{1}} x\right) .
$$

For each $x \in X$ and $\mathbf{j}=\left(j_{1}, j_{2}, \ldots\right) \in \Sigma$ we define

$$
Z_{n}(x, \mathbf{j})=T_{j_{n}} \cdots T_{j_{1}}(x)
$$

and set $Z_{0}(x, \mathbf{j})=x$. Then $Z_{n}(x, \mathbf{j})$ is an $X$-valued Markov chain with respect to $\mu_{x}$, with initial state $x$ and transition probability $p$. For convenience if $\mathbf{j}=\left(j_{1}, j_{2}, \ldots\right) \in \Sigma$ then we shall often write $T_{n}(\mathbf{j})=T_{j_{n}} \cdots T_{j_{1}}$.

We can relate $\mu_{x}$ and $\nu$ as follows [E2]. Define $\pi_{x}(\mathbf{j})=\lim _{n \rightarrow \infty} T_{j_{1}} T_{j_{2}} \cdots T_{j_{n}}(x)$ for $\mu_{x}$-a.e. $\mathbf{j} \in \Sigma$. Then for all $x \in X$ we have $\pi_{x}^{*} \mu_{x}=\nu$.

Let $f: X \rightarrow \mathbb{R}$ be a continuous function on $X$. We are interested in the distribution of the sequence of observations

$$
S_{n} f(x, \mathbf{j})=\sum_{k=1}^{n} f\left(Z_{k}(x, \mathbf{j})\right) .
$$

It is known [E1] that $S_{n} f$ satisfies a pointwise ergodic theorem: for all $x \in X$ and $\mu_{x}$-a.e. $\mathbf{j} \in \Sigma$,

$$
\lim _{n \rightarrow \infty} \frac{1}{n} S_{n} f(x, \mathbf{j})=\nu(f) .
$$

Under the mild technical hypotheses stated in $\S 3.1$, a central limit theorem is also known to hold [Pe, HH2]. Let $f: X \rightarrow \mathbb{R}$ be a bounded Lipschitz function and fix $x \in X$. Then

$$
\frac{1}{\sqrt{n}} S_{n} f(x, \cdot) \rightarrow_{d} \mathcal{N}_{\nu(f), \sigma^{2}(f)},
$$


provided that the variance $\sigma^{2}(f)>0$. Here $\mathcal{N}_{\nu(f), \sigma^{2}(f)}$ denotes the normal distribution with mean $\nu(f)$ and variance $\sigma^{2}(f)$ and $\rightarrow_{d}$ denotes convergence in distribution. The variance is given by

$$
\sigma^{2}(f)=\lim _{n \rightarrow \infty} \frac{1}{n} \int\left(S_{n} f(x, \cdot)-n \nu(f)\right)^{2} d \mu_{x} .
$$

If $f$ is a bounded Lipschitz function then $\sigma^{2}(f)<\infty$.

The space $X$ is typically not compact and so it makes sense to consider functions $f$ which satisfy some degree of regularity (a Hölder condition, for example) but which are not in $L^{2}$ and which do not have a finite variance. In this case, it is natural to conjecture that the sequence of observations (4), when normalised by a sequence that grows like $n^{1 / p}$ for a suitable parameter $p \in(0,2)$ (called the order), converges in distribution to a stable distribution $Y_{p, \beta, b, c}$ (where $p \in(0,2), \beta \in[-1,1], b \in \mathbb{R}, c>0$ are parameters described in $\S 5)$. Stable laws can be characterised as being generalisations of the Gaussian distribution that keep the stability property: if $X$ and $Y$ are two random variables with the same stable distribution (up to an affine rescaling) then $X+Y$ has the same distribution (up to an affine rescaling). Stable laws of order $p=1$ are technically more difficult to deal with [AD1] and for simplicity we concentrate on the case $p \in(0,1) \cup(1,2)$. We give a brief introduction to stable laws and their properties that we shall need in $\S 5$.

Let $f: X \rightarrow \mathbb{R} \in L^{1}(\nu)$ be continuous. We assume that $f$ satisfies a Hölder condition that we make precise in $\S 3.2$. Assume in addition that for some $p \in(0,2)$

$$
\nu\{x \mid f(x)>t\}=\frac{1}{t^{p}}\left(C_{1}+o(1)\right), \quad \nu\{x \mid f(x)<-t\}=\frac{1}{t^{p}}\left(C_{2}+o(1)\right),
$$

for constants $C_{1}, C_{2}>0$. (This condition can be weakened to include a slowly varying function - see $§ \S 5,7,8$.)

Our main result is the stable limit theorem.

Theorem 2.1 (Distributional Stable Limit Theorem)

Suppose that the IFS $\left(T_{j}, p_{j}\right)$ contracts on average and satisfies the technical hypotheses in $\S 3.1$. Suppose that $f: X \rightarrow \mathbb{R}$ satisfies a Hölder condition stated in $\S 3.2$ and that, for some $p \in(0,2)$, (7) holds. Then for all $x \in X$,

$$
\mu_{x}\left\{\mathbf{j} \in \Sigma \mid \frac{S_{n} f(x, \mathbf{j})-a_{n}}{n^{1 / p}}<t\right\} \rightarrow \int_{-\infty}^{t} d Y_{p}
$$

as $n \rightarrow \infty$, for some stable law $Y_{p}$ of order $p$, where

$$
a_{n}= \begin{cases}0 & \text { if } p<1 \\ n \nu(f), & \text { if } p>1\end{cases}
$$


Given an observation $f$, the key to our results is an analysis of the spectral properties of a one-parameter family of transfer operators $P_{t}$ acting on a certain Banach space of functions. The expansion of the maximal eigenvalue $\lambda_{t}$ of $P_{t}$ as a function of $t$ is intimately related to the characteristic function of the respective stable distribution. An observation $f$ that essentially does not take values in a lattice will produce a non-periodic perturbation of the spectrum of the transfer operator $P$. In this case the only $t$ for which $P_{t}$ has an eigenvalue of modulus 1 is $t=0$. Lattice-valued observations $f$ give rise to periodicity in $t$ of the spectrum of $P_{t}$.

For a non-arithmetic observation $f$ we have the following local limit theorem.

Theorem 2.2 (Non-arithmetic local stable limit theorem)

Suppose that the IFS $\left(T_{j}, p_{j}\right)$ contracts on average and satisfies the technical hypotheses in $\S 3.1$. Suppose that $f: X \rightarrow \mathbb{R}$ satisfies a Hölder condition given in $\S 3.2$ and the non-arithmeticity assumption in $\S 8.1$, and that, for some $p \in(0,2),(7)$ holds. Then there exists a stable law $Y_{p}$ of order $p$ with density $y_{p}$ such that for any $a, b \in \mathbb{R}, a<b$ and any $x \in X$ we have

$$
\lim _{n \rightarrow \infty}\left|n^{1 / p} \mu_{x}\left\{\mathbf{j} \in \Sigma \mid S_{n} f(x, \mathbf{j})-a_{n} \in z+[a, b]\right\}-y_{p}\left(z / n^{1 / p}\right)(b-a)\right|=0
$$

uniformly in $z \in \mathbb{R}$, where $a_{n}$ is given by (8).

In the case where the observation $f$ has finite variance, the same method of proof also provides a local central limit theorem.

Theorem 2.3 (Non-arithmetic local central limit theorem)

Suppose that the IFS $\left(T_{j}, p_{j}\right)$ contracts on average and satisfies the technical hypotheses in $\S 3.1$. Suppose that $f: X \rightarrow \mathbb{R}$ satisfies a Hölder condition given in $\S 3.2$ and the non-arithmeticity assumption in $\S 8.1$, and $0<\sigma^{2}(f)<$ $\infty$. Then for any $a, b \in \mathbb{R}, a<b$ and any $x \in X$ we have

$$
\begin{aligned}
\lim _{n \rightarrow \infty} & \mid\left(2 \pi \sigma^{2}(f) n\right)^{1 / 2} \mu_{x}\left\{\mathbf{j} \in \Sigma \mid S_{n} f(x, \mathbf{j})-n \nu(f) \in z+[a, b]\right\} \\
& -e^{\frac{-z^{2}}{2 \sigma^{2}(f) n}}(b-a) \mid=0
\end{aligned}
$$

uniformly in $z \in \mathbb{R}$.

\section{$\S 3 \quad$ Assumptions and function spaces}

\section{$\S 3.1$ Technical hypotheses}

Let $(X, d)$ be a locally compact second countable metric space. Choose and fix a choice of origin $x_{0} \in X$. 
Let $T_{j}: X \rightarrow X$ be a finite or countable family of Lipschitz maps. If $T: X \rightarrow X$ is Lipschitz then we define

$$
\|T\|=\sup _{x, y \in X, x \neq y} \frac{d(T x, T y)}{d(x, y)} .
$$

Let $p_{j}: X \rightarrow[0,1]$ be a countable family of probability functions such that $p_{j}(x) \geq 0$ for all $x \in X, j \in \mathbb{N}$ and are Dini continuous. Define the probability measure $\mu_{x}$ on $\Sigma$ by (3). Define $m\left(p_{j}\right)=\sup \left\{\left|p_{j}(x)-p_{j}(y)\right| \mid\right.$ $x, y \in X, d(x, y) \leq 1\}$.

Definition. The IFS contracts on average after $n_{0}$ steps if there exists $r \in(0,1)$ such that

$$
\sup _{x} \mathbb{E}_{x}\left(\left\|T_{n_{0}}(\mathbf{j})\right\|\right) \leq r .
$$

We remark that there exist examples of IFSs $\left(T_{j}, p_{j}\right)$ that contract on average after $n_{0}$ steps, but which do not contract after 1 step and for which none of the $T_{j}$ are strict contractions $[\mathrm{Pe}]$.

We will assume the following technical conditions hold.

(i) We have

$$
\sup _{x, y \in X} \mathbb{E}_{x}\left(\frac{d\left(T_{j}(y), x_{0}\right)}{1+d\left(y, x_{0}\right)}\right)<\infty .
$$

(ii) We have

$$
\sup _{x \in X} \mathbb{E}_{x}\left(\max \left\{1,\left\|T_{j}\right\|\right\}+d\left(T_{j} x_{0}, x_{0}\right)\right) m\left(p_{j}\right)<\infty .
$$

(iii) We assume that for each $x, y \in X$ there exists $\mathbf{i}=\left(i_{1}, i_{2}, \ldots\right) \in \Sigma$ such that for each $m$,

$$
\mu_{x}\left[i_{1}, i_{2}, \ldots, i_{m}\right], \mu_{y}\left[i_{1}, i_{2}, \ldots, i_{m}\right] \neq 0 .
$$

Assumption (10) can be viewed as a moment assumption on the $T_{j}$. Note that (10), (11) are automatically satisfied if there are finitely many maps. Assumption (12) is an irreducibility assumption that ensures that the transfer operator below has 1 as the unique simple maximal eigenvalue.

\section{$\S 3.2 \quad$ Function spaces}

We assume that the continuous observation $f: X \rightarrow \mathbb{R}$ satisfies a uniform Hölder condition, namely for some $\alpha \in(0,1]$ :

$$
|f|_{(\alpha)}=\sup _{x, y \in X, x \neq y} \frac{|f(x)-f(y)|}{d(x, y)^{\alpha}}<\infty .
$$


For example, if $X=\mathbb{R}$ then $f(x)=x^{\alpha}$ satisfies (13). In this case, $\sigma^{2}(f)=\infty$ if $2 \alpha>\theta_{0}$.

Choose $\varepsilon, \beta, \gamma>0$ such that

$$
0<\varepsilon<\varepsilon+\beta<\gamma<\varepsilon+2 \beta<1 .
$$

For $\lambda \in[0,1]$ we define $d_{\lambda}(x)=1+\lambda d\left(x, x_{0}\right)$.

We will fix a choice of $\lambda$ below. For a continuous function $w: X \rightarrow \mathbb{C}$ define

$$
|w|_{\gamma}=\sup _{x \in X} \frac{|w(x)|}{d_{\lambda}(x)^{\gamma}}
$$

and

$$
|w|_{\varepsilon, \beta}=\sup _{x, y \in X, x \neq y} \frac{|w(x)-w(y)|}{d(x, y)^{\varepsilon} d_{\lambda}(x)^{\beta} d_{\lambda}(y)^{\beta}} .
$$

Then $|\cdot|_{\gamma}$ is a norm and $|w|_{\varepsilon, \beta}$ is a semi-norm. We define a norm by

$$
\|w\|_{\varepsilon, \beta, \gamma}=|w|_{\gamma}+|w|_{\varepsilon, \beta} .
$$

Then the spaces $C_{\gamma}=\left\{w: X \rightarrow \mathbb{R} \mid w\right.$ is continuous and $\left.|w|_{\gamma}<\infty\right\}$ and $C_{\varepsilon, \beta, \gamma}=\left\{w \in C_{\gamma} \mid\|w\|_{\varepsilon, \beta, \gamma}<\infty\right\}$ are Banach spaces with respect to the norms $|\cdot|_{\gamma}$ and $\|\cdot\|_{\varepsilon, \beta, \gamma}$, respectively. Such function spaces have been wellused in the study of IFSs and random walks on groups [GP, HH1, HH2, Pe]. Note that if $\lambda_{1}, \lambda_{2} \in(0,1]$ then $d_{\lambda_{1}}(x) \leq \lambda_{2}^{-1} d_{\lambda_{2}}(x)$. Although different values of $\lambda \in(0,1)$ give different norms $|\cdot|_{\gamma}$ and $\|\cdot\|_{\varepsilon, \beta, \gamma}$, it follows that the norms are equivalent.

Let $T: X \rightarrow X$ be Lipschitz. Define

$$
\delta_{\lambda}(T)=\sup _{x \in X} \frac{d_{\lambda}(T x)}{d_{\lambda}(x)} .
$$

\section{Lemma 3.1}

If $T: X \rightarrow X$ is Lipschitz then $\delta_{\lambda}(T) \leq \max \{1,\|T\|\}+\lambda d\left(T x_{0}, x_{0}\right)$.

Proof. Observe that

$$
\begin{aligned}
\frac{d_{\lambda}(T x)}{d_{\lambda}(x)} & \leq \frac{1+\lambda d\left(T x, T x_{0}\right)}{1+\lambda d\left(x, x_{0}\right)}+\frac{\lambda d\left(T x_{0}, x_{0}\right)}{1+\lambda d\left(x, x_{0}\right)} \\
& \leq \frac{1+\lambda\|T\| d\left(x, x_{0}\right)}{1+\lambda d\left(x, x_{0}\right)}+\lambda d\left(T x_{0}, x_{0}\right) \\
& \leq \max \{1,\|T\|\}+\lambda d\left(T x_{0}, x_{0}\right) .
\end{aligned}
$$


We now choose $\lambda$. Note that, as $\varepsilon+2 \beta<1$ and taking $n=q n_{0}$, it follows from (9) that

$$
\begin{aligned}
\sup _{x} & \mathbb{E}_{x}\left(\left\|T_{q n}(\cdot)\right\|^{\varepsilon}\left(1+\left\|T_{q n}(\cdot)\right\|\right)^{\varepsilon+2 \beta}\right) \\
\leq & \sup _{x} \mathbb{E}_{x}\left(\left\|T_{q n}(\cdot)\right\|^{\varepsilon}\right)+\sup _{x} \mathbb{E}_{x}\left(\left\|T_{q n}(\cdot)\right\|^{\varepsilon+2 \beta}\right) \\
\leq & r^{q \varepsilon}+r^{q(\varepsilon+2 \beta)} .
\end{aligned}
$$

By fixing $q$ sufficiently large we can ensure that $r^{q \varepsilon}+r^{q(\varepsilon+2 \beta)}<1$. Let $n_{0}^{\prime}=$ $q n_{0}$ for this value of $q$. As $d_{\lambda}\left(T_{n}(\mathbf{j})\right)^{2 \beta}<1+\left\|T_{n}(\mathbf{j})\right\|^{2 \beta}+\lambda^{2 \beta} d\left(T_{n}(\mathbf{j}) x_{0}, x_{0}\right)^{2 \beta}$, by choosing $\lambda$ sufficiently small we can ensure that

$$
\sup _{x} \mathbb{E}_{x}\left(\left\|T_{n_{0}^{\prime}}(\cdot)\right\|^{\varepsilon} d_{\lambda}\left(T_{n_{0}^{\prime}}(\cdot)\right)^{2 \beta}\right)<r^{\prime}<1
$$

for some $r^{\prime}<1$. We now fix $\lambda$ as in (15).

The following result is well-known.

\section{Lemma 3.2}

The space $C_{\varepsilon, \beta, \gamma}$ is a Banach space with respect to the norm $\|\cdot\|_{\varepsilon, \beta, \gamma}$.

Motivated by [HH1, HH2], we introduce the following norm: for $w \in$ $C_{\varepsilon, \beta, \gamma}$ define

$$
\|w\|_{\varepsilon, \beta}^{(1)}=\int|w| d \nu+|w|_{\varepsilon, \beta} .
$$

We shall write $|w|^{(1)}=\int|w| d \nu$.

\section{Lemma 3.3}

The space $C_{\varepsilon, \beta, \gamma}$ is a Banach space when equipped with the norm $\|\cdot\|_{\varepsilon, \beta}^{(1)}$. Moreover, the norms $\|\cdot\|_{\varepsilon, \beta}^{(1)}$ and $\|\cdot\|_{\varepsilon, \beta, \gamma}$ are equivalent.

Proof. We prove that $C_{\varepsilon, \beta, \gamma}$ is a Banach space with respect to $\|\cdot\|_{\varepsilon, \beta}^{(1)}$. Let $w_{n} \in C_{\varepsilon, \beta, \gamma}$ be a Cauchy sequence with respect to $\|\cdot\|_{\varepsilon, \beta}^{(1)}$. Let $y_{0} \in X$ and define $v_{n}(x)=w_{n}(x)-w_{n}\left(y_{0}\right)$. Then

$$
\begin{aligned}
\left|v_{n}(x)-v_{m}(x)\right| & =\left|\left(w_{n}(x)-w_{m}(x)\right)-\left(w_{n}\left(y_{0}\right)-w_{m}\left(y_{0}\right)\right)\right| \\
& \leq\left|w_{n}-w_{m}\right|_{\varepsilon, \beta} d\left(x, y_{0}\right)^{\varepsilon} d_{\lambda} x^{\beta} d_{\lambda}\left(y_{0}\right)^{\beta}
\end{aligned}
$$

so that

$$
\nu\left(\left|v_{n}-v_{m}\right|\right) \leq\left|w_{n}-w_{m}\right|_{\varepsilon, \beta} d_{\lambda}\left(y_{0}\right)^{\beta} \int d\left(x, y_{0}\right)^{\varepsilon} d_{\lambda}\left(x, x_{0}\right) d \nu
$$

As the integrand in (16) is $O\left(d\left(x, y_{0}\right)^{\varepsilon+\beta}\right)$ and $\varepsilon+\beta<\theta_{0}$, it follows that $\nu\left(\left|v_{n}-v_{m}\right|\right) \leq C\left|w_{n}-w_{m}\right|_{\varepsilon, \beta}$ for some constant $C>0$. Hence $v_{n}$ is a 
Cauchy sequence in the Banach space $L^{1}(\nu)$ of $L^{1}$ functions with respect to $\nu$. Hence $v_{n}$ converges in $L^{1}(\nu)$ to, say, $v \in L^{1}(\nu)$ and $\nu\left(v_{n}\right) \rightarrow \nu(v)$.

As $w_{n}$ is a Cauchy sequence with respect to $\|\cdot\|_{\varepsilon, \beta}^{(1)}$, we see that $\nu\left(w_{n}\right)$ is a Cauchy sequence of complex numbers, and so converges. Note that for all $x, w_{n}\left(y_{0}\right)=w_{n}(x)-w_{n}(x)$. Integrating this with respect to $\nu$ we obtain $w_{n}\left(y_{0}\right)=\nu\left(w_{n}\right)-\nu\left(v_{n}\right)$. Hence for each $y_{0}, w_{n}\left(y_{0}\right)$ converges. Hence $w_{n}$ converges pointwise to some function, say $w$. As $\nu\left(w_{n}\right)$ converges, it follows from the Dominated Convergence Theorem that $\nu\left(w_{n}\right) \rightarrow \nu(w)$.

It remains to check that $w \in C_{\varepsilon, \beta, \gamma}$. Given $\epsilon>0$, choose $N$ such that if $n, m \geq N$ then $\left|w_{n}-w_{m}\right|_{\varepsilon, \beta}<\epsilon$. Letting $m \rightarrow \infty$ implies that $\left|w_{n}-w\right|_{\varepsilon, \beta} \leq$ $\epsilon$. Also note that

$$
\left|w_{n}(x)-w_{n}\left(x_{0}\right)\right| \leq\left|w_{n}\right|_{\varepsilon, \beta} d\left(x, x_{0}\right)^{\varepsilon} d_{\lambda}(x)^{\beta}
$$

letting $n \rightarrow \infty$ and dividing by $d_{\lambda}(x)^{\gamma}$ it follows that

$$
\frac{|w(x)|}{d_{\lambda}(x)^{\gamma}} \leq \frac{\left|w\left(x_{0}\right)\right|}{d_{\lambda}(x)^{\gamma}}+|w|_{\varepsilon, \beta} \frac{d\left(x, x_{0}\right)^{\varepsilon} d_{\lambda}(x)^{\beta}}{d_{\lambda}(x)^{\gamma}} .
$$

As $\varepsilon+\beta<\gamma$, we have that $d\left(x, x_{0}\right)^{\varepsilon} d_{\lambda}(x)^{\beta} / d_{\lambda}(x)^{\gamma} \leq C$, for some constant $C>0$. Hence $|w|_{\gamma}<\infty$.

We prove that the two norms $\|\cdot\|_{\varepsilon, \beta, \gamma},\|\cdot\|_{\varepsilon, \beta}^{(1)}$ on $C_{\varepsilon, \beta, \gamma}$ are equivalent. For $w \in C_{\varepsilon, \beta, \gamma}$ note that

$$
\begin{aligned}
|w(y)| \leq & |w(x)|+|w|_{\varepsilon, \beta} d(x, y)^{\varepsilon} d_{\lambda}(x)^{\beta} d_{\lambda}(y)^{\beta} \\
\leq & |w(x)|+|w|_{\varepsilon, \beta} d(x, y)^{\varepsilon}\left(d\left(x, x_{0}\right)^{\varepsilon} d_{\lambda}(x)^{\beta} d_{\lambda}(y)^{\beta}\right. \\
& \left.+d\left(y, x_{0}\right)^{\varepsilon} d_{\lambda}(x)^{\beta} d_{\lambda}(y)^{\beta}\right) .
\end{aligned}
$$

Hence there exists a constant $C>0$ such that

$$
|w(y)| \leq \nu(|w|)+|w|_{\varepsilon, \beta} d_{\lambda}(y)^{\beta} C\left(\nu\left(d\left(x, x_{0}\right)^{\varepsilon+\beta}+d\left(x, x_{0}\right)^{\beta}\right)\right) .
$$

As $\beta<\gamma$, it follows that

$$
\frac{|w(y)|}{d_{\lambda}(y)^{\gamma}} \leq \frac{|w(y)|}{d_{\lambda}(y)^{\beta}} \leq \nu(|w|)+C^{\prime}|w|_{\varepsilon, \beta}
$$

for some constant $C^{\prime}>0$. Hence $\|w\|_{\varepsilon, \beta, \gamma} \leq \nu(|w|)+\left(C^{\prime}+1\right)|w|_{\varepsilon, \beta} \leq$ $\left(C^{\prime}+1\right)\|w\|_{\varepsilon, \beta}^{(1)}$.

As $C_{\varepsilon, \beta, \gamma}$ is a Banach space with respect to both $\|\cdot\|_{\varepsilon, \beta, \gamma}$ and $\|\cdot\|_{\varepsilon, \beta}^{(1)}$ it follows from the Open Mapping Theorem that there exists a constant $C^{\prime \prime}>0$ such that $\|w\|_{\varepsilon, \beta}^{(1)} \leq C^{\prime \prime}\|w\|_{\varepsilon, \beta, \gamma}$.

We shall need the following result. 


\section{Lemma 3.4}

The inclusion $\iota:\left(C_{\varepsilon, \beta, \gamma},\|\cdot\|_{\varepsilon, \beta}^{(1)}\right) \hookrightarrow\left(C_{\varepsilon, \beta, \gamma},|\cdot|^{(1)}\right)$ is compact.

Proof. Let $w_{n} \in C_{\varepsilon, \beta, \gamma}$ and suppose that $\left\|w_{n}\right\|_{\varepsilon, \beta}^{(1)} \leq 1$. As $\left|w_{n}\right|_{\varepsilon, \beta}<1$, it follows that $w_{n}$ is equicontinuous on every compact subset of $X$. By a diagonalisation argument, there exists a subsequence $n_{k} \rightarrow \infty$ such that $w_{n_{k}}$ converges uniformly to $w \in C_{\varepsilon, \beta, \gamma},\|w\|_{\varepsilon, \beta}^{(1)} \leq 1$, on every compact set. As $|w(x)| \leq d_{\lambda}(x)^{\gamma}$, it follows from the Dominated Convergence Theorem that $\left|w-w_{n_{k}}\right|^{(1)}=\nu\left(\left|w_{n_{k}}-w\right|\right) \rightarrow 0$.

\section{$\S 4$ Spectral properties of a family of transfer operators}

Define the operator $P$ on continuous functions $w$ by

$$
P w(x)=\sum_{j} p_{j}(x) w\left(T_{j} x\right)
$$

Then $P$ maps the space of continuous functions to itself and $P 1=1$, so that 1 is an eigenvalue for $P$. For stronger spectral properties of $P$ to hold we need to restrict $P$ to $C_{\varepsilon, \beta, \gamma}$. The following result is proved in [Pe], albeit on a slightly different function space; we sketch the argument in $\S 4.1$ below.

\section{Proposition 4.1 ([Pe])}

Under the technical hypotheses in $\S 3.1$, the operator $P$ maps $C_{\varepsilon, \beta, \gamma}$ to itself, has 1 as a simple maximal eigenvalue with associated eigenprojection $\nu$, and the remainder of the spectrum is contained within a disc of radius $\rho<1$.

We will need to study the spectral properties of the following oneparameter family of perturbed transfer operators. Fix a continuous function $f$ with $|f|_{(\alpha)}<\infty$. For each $t \in \mathbb{R}$ define

$$
P_{t} w=P\left(e^{i t f} w\right)=\sum_{j} p_{j}(x) e^{i t f\left(T_{j} x\right)} w\left(T_{j} x\right) .
$$

We shall see below that $P_{t}$ maps $C_{\varepsilon, \beta, \gamma}$ to itself. The relevance of $P_{t}$ to the sums of observations (4) is given by observing that

$$
P_{t}^{n} w(x)=\mathbb{E}_{x}\left(e^{i t S_{n} f(x, \cdot)} w\left(Z_{n}(x, \cdot)\right)\right) .
$$

We will prove that, for sufficiently small $t, P_{t}$ has a unique simple maximal eigenvalue $\lambda_{t}$ with corresponding eigenprojection $\pi_{t}$ and a spectral gap so that the remainder of the spectrum is contained within a disc of radius $\rho_{t}$. We will do this by establishing a Lasota-Yorke inequality for $P_{t}$ and citing a result of Hennion $[\mathrm{H}]$. We also want to determine the continuity properties of $\lambda_{t}, \pi_{t}, \rho_{t}$, etc, as $t$ varies. To do this, we will apply a theorem of Keller and Liverani [KL]. 


\section{$\S 4.1 \quad$ A Lasota-Yorke inequality}

We shall need the following estimates.

\section{Lemma 4.2}

(i) Let $\beta \in(0,1)$. Then

$$
B_{n}(\beta)=\sup _{x, y} \mathbb{E}_{x}\left(\frac{d_{\lambda}\left(Z_{n}(y, \cdot)\right)^{\beta}}{d_{\lambda}(y)^{\beta}}\right)<\infty .
$$

(ii) We have

$$
A(\gamma, \varepsilon)=\sup _{x, y \in X, x \neq y} \sum_{j} \frac{d_{\lambda}\left(T_{j} x\right)^{\gamma}}{d_{\lambda}(x)^{\gamma}} \frac{\left|p_{j}(x)-p_{j}(y)\right|}{d(x, y)^{\varepsilon}}<\infty .
$$

Proof. We prove (i). Let $n_{0}$ be as in (9) and let

$$
R=\sup _{\ell=0, \ldots, n_{0}-1} \sup _{x, y, z, y \neq z} \mathbb{E}_{x}\left(\frac{d\left(Z_{\ell}(y, \cdot), Z_{\ell}(z, \cdot)\right)}{d(y, z)}\right)<\infty .
$$

Then $\mathbb{E}_{x}\left(d\left(Z_{n}(y, \cdot), Z_{n}(z, \cdot)\right)\right) \leq R r^{\left\lfloor\frac{n}{n_{0}}\right\rfloor}=R \rho^{n}$ for some $\rho \in(0,1)$, increasing $R$ slightly if necessary. Then

$$
\begin{aligned}
& \mathbb{E}_{x}\left(d_{\lambda}\left(Z_{k}(y, \cdot)\right)^{\beta}\right) \\
& \quad \leq 1+\lambda^{\beta} \mathbb{E}_{x}\left(d\left(Z_{k}(y, \cdot), Z_{k}\left(x_{0}, \cdot\right)\right)\right)^{\beta}+\lambda^{\beta} \mathbb{E}_{x}\left(d\left(Z_{k}\left(x_{0}, \cdot\right), x_{0}\right)\right)^{\beta} .
\end{aligned}
$$

Now

$$
\begin{aligned}
& \mathbb{E}_{x}\left(d\left(Z_{k}\left(x_{0}, \cdot\right), x_{0}\right)\right)^{\beta} \\
& \leq \sum_{\ell=1}^{k} \sum_{j_{\ell}, \cdots, j_{k}} \mu_{T_{j_{\ell}-1} \cdots T_{j_{1}}\left(x_{0}\right)}\left[j_{\ell}, \ldots, j_{k}\right] d\left(T_{j_{k}} \cdots T_{j_{\ell+1}}\left(T_{\ell} x_{0}\right), T_{j_{k}} \cdots T_{j_{\ell+1}}\left(x_{0}\right)\right)^{\beta} \\
& \leq \sum_{\ell=1}^{k} R \rho^{k-\ell} \sup _{x} \mathbb{E}_{x} d\left(Z_{1}\left(x_{0}, \cdot\right), x_{0}\right),
\end{aligned}
$$

which is bounded by a constant, by (10). The middle term in the right-hand side of (18) is bounded by $\lambda^{\beta} R^{\beta} \rho^{n \beta} d\left(y, x_{0}\right)^{\beta}$. It follows that $B_{n}(\beta)<\infty$.

We prove (ii). If $d(x, y) \geq 1$ then $1 / d(x, y) \leq 1$ so that

$$
\begin{aligned}
A(\gamma, \varepsilon) & \leq \sup _{x, y \in X, x \neq y} \sum_{j} \frac{d_{\lambda}\left(T_{j} x\right)^{\gamma}}{d_{\lambda}(x)^{\gamma}} p_{j}(x)+\frac{d_{\lambda}\left(T_{j} x\right)^{\gamma}}{d_{\lambda}(x)^{\gamma}} p_{j}(y) \\
& \leq \sup _{x, y} \mathbb{E}_{x}\left(\delta\left(T_{j}\right)^{\gamma}\right)+\mathbb{E}_{y}\left(\delta\left(T_{j}\right)^{\gamma}\right) \\
& \leq 2 B_{1}(\gamma) .
\end{aligned}
$$


If $d(x, y) \leq 1$ then, recalling that $m\left(p_{j}\right)=\sup _{x, y \in X, d(x, y) \leq 1}\left|p_{j}(x)-p_{j}(y)\right| / d(x, y)$, we have

$$
\begin{aligned}
\sum_{j} \frac{d_{\lambda}\left(T_{j} x\right)^{\gamma}}{d_{\lambda}(x)^{\gamma}} \frac{\left|p_{j}(x)-p_{j}(y)\right|}{d(x, y)^{\varepsilon}} & =\sum_{j} \frac{d_{\lambda}\left(T_{j} x\right)^{\gamma}}{d_{\lambda}(x)^{\gamma}} \frac{\left|p_{j}(x)-p_{j}(y)\right|}{d(x, y)} d(x, y)^{1-\varepsilon} \\
& \leq \sum_{j} \delta_{\lambda}\left(T_{j}\right)^{\gamma} m\left(p_{j}\right) .
\end{aligned}
$$

Hence (ii) follows from (i) and (11).

We can now prove a Lasota-Yorke inequality for $P_{t}$.

\section{Proposition 4.3}

There exist constants $R_{n}>0$ such that for all $w \in C_{\varepsilon, \beta, \gamma}$ we have

$$
\left|P_{t}^{n} w\right|_{\varepsilon, \beta} \leq \sup _{x} \mathbb{E}_{x}\left(\left\|T_{n}(\cdot)\right\|^{\varepsilon} \delta_{\lambda}\left(T_{n}(\cdot)\right)^{2 \beta}\right)|w|_{\varepsilon, \beta}+R_{n}|t|^{\varepsilon / \alpha}|w|_{\gamma}
$$

Proof. Let $w \in C_{\varepsilon, \beta, \gamma}$.

First note that

$$
\begin{aligned}
\left|P_{t}^{n} w(x)\right| & \leq \sum_{j_{1}, \ldots, j_{n}} \mu_{x}\left[j_{1}, \ldots, j_{n}\right]\left|w\left(Z_{n}(x, \mathbf{j})\right)\right| \\
& \leq \sum_{j_{1}, \ldots, j_{n}} \mu_{x}\left[j_{1}, \ldots, j_{n}\right]|w|_{\gamma} d_{\lambda}\left(Z_{n}(x, \mathbf{j})\right)^{\gamma} \\
& \leq \sum_{j_{1}, \ldots, j_{n}} \mu_{x}\left[j_{1}, \ldots, j_{n}\right]|w|_{\gamma} \delta_{\lambda}\left(T_{n}(\mathbf{j})\right)^{\gamma} d_{\lambda}(x)^{\gamma}
\end{aligned}
$$

so that

$$
\left|P_{t}^{n} w\right|_{\gamma} \leq \mathbb{E}_{x}\left(\delta_{\lambda}\left(T_{n}(\cdot)\right)^{\gamma}\right)|w|_{\gamma}
$$

Note that

$$
\mathbb{E}_{x}\left(\delta_{\lambda}\left(T_{n}(\cdot)\right)^{\gamma}\right) \leq 1+\mathbb{E}_{x}\left(\left\|T_{n}(\cdot)\right\|\right)^{\gamma}+\lambda^{\gamma} \mathbb{E}_{x}\left(d\left(T_{j} x_{0}, x_{0}\right)^{\gamma}\right)<\infty
$$

as $\gamma<1$ and (10). Hence there exists $M>0$ such that $\left|P_{t}^{n}\right|_{\gamma} \leq M^{n}$.

Let $x, y \in X$ and assume, without loss of generality, that $d_{\lambda}(y) \leq d_{\lambda}(x)$.

We can write

$$
\begin{aligned}
& \left|P_{t}^{n} w(x)-P_{t}^{n} w(y)\right| \\
& \quad=\left|\sum_{j_{1}, \ldots, j_{n}}\left(\mu_{x}\left[j_{1}, \ldots, j_{n}\right] e^{i t S_{n} f(x, \mathbf{j})} w\left(Z_{n}(x, \mathbf{j})\right)-\mu_{y}\left[j_{1}, \ldots, j_{n}\right] e^{i t S_{n} f(y, \mathbf{j})} w\left(Z_{n}(y, \mathbf{j})\right)\right)\right| \\
& \quad \leq \Sigma_{w}^{(n)}+\Sigma_{f}^{n}+\Sigma_{\mu}^{n}
\end{aligned}
$$


where

$$
\begin{aligned}
\Sigma_{w}^{(n)} & =\left|\sum_{j_{1}, \ldots, j_{n}} \mu_{x}\left[j_{1}, \ldots, j_{n}\right] e^{i t S_{n} f(x, \mathbf{j})}\left(w\left(Z_{n}(x, \mathbf{j})\right)-w\left(Z_{n}(y, \mathbf{j})\right)\right)\right|, \\
\Sigma_{f}^{(n)} & =\left|\sum_{j_{1}, \ldots, j_{n}} \mu_{x}\left[j_{1}, \ldots, j_{n}\right]\left(e^{i t S_{n} f(x, \mathbf{j})}-e^{i t S_{n}(y, \mathbf{j})}\right) w\left(Z_{n}(y, \mathbf{j})\right)\right|, \\
\Sigma_{\mu}^{(n)} & =\left|\sum_{j_{1}, \ldots, j_{n}}\left(\mu_{x}\left[j_{1}, \ldots, j_{n}\right]-\mu_{y}\left[j_{1}, \ldots, j_{n}\right]\right) e^{i t S_{n} f(y, \mathbf{j})} w\left(Z_{n}(y, \mathbf{j})\right)\right| .
\end{aligned}
$$

Now

$$
\begin{aligned}
\Sigma_{w}^{(n)} & \leq \sum_{j_{1}, \ldots, j_{n}} \mu_{x}\left[j_{1}, \ldots, j_{n}\right]\left|w\left(Z_{n}(x, \mathbf{j})\right)-w\left(Z_{n}(y, \mathbf{j})\right)\right| \\
& \leq \sum_{j_{1}, \ldots, j_{n}} \mu_{x}\left[j_{1}, \ldots, j_{n}\right]|w|_{\varepsilon, \beta} d\left(Z_{n}(x, \mathbf{j}), Z_{n}(y, \mathbf{j})\right)^{\varepsilon} d_{\lambda}\left(Z_{n}(x, \mathbf{j})\right)^{\beta} d_{\lambda}\left(Z_{n}(y, \mathbf{j})\right)^{\beta} \\
& \leq|w|_{\varepsilon, \beta} \mathbb{E}_{x}\left(\left\|T_{n}(\cdot)\right\|^{\varepsilon} \delta_{\lambda}\left(T_{n}(\cdot)\right)^{2 \beta}\right) d(x, y)^{\varepsilon} d_{\lambda}(x)^{\beta} d_{\lambda}(y)^{\beta}
\end{aligned}
$$

We can write

$$
\begin{aligned}
\Sigma_{f}^{(n)} & \leq \sum_{j_{1}, \ldots, j_{n}} \mu_{x}\left[j_{1}, \ldots, j_{n}\right]\left|e^{i t S_{n} f(x, \mathbf{j})}-e^{i t S_{n} f(y, \mathbf{j})}\right|\left|w\left(Z_{n}(x, \mathbf{j})\right)\right| \\
& \leq|w|_{\gamma} \sum_{j_{1}, \ldots, j_{n}} \mu_{x}\left[j_{1}, \ldots, j_{n}\right]\left|e^{i t\left(S_{n} f(x, \mathbf{j})-S_{n} f(y, \mathbf{j})\right)}-1\right| d_{\lambda}\left(Z_{n}(y, \mathbf{j})\right)^{\gamma} \\
& \leq|w|_{\gamma} \sum_{k=1}^{n} \Sigma_{f}^{(n), k}
\end{aligned}
$$

where

$$
\Sigma_{f}^{(n), k}=\sum_{j_{1}, \ldots, j_{n}} \mu_{x}\left[j_{1}, \ldots, j_{n}\right]\left|e^{i t\left(f\left(Z_{k}(x, \mathbf{j})\right)-f\left(Z_{k}(y, \mathbf{j})\right)\right)}-1\right| d_{\lambda}\left(Z_{n}(y, \mathbf{j})\right)^{\gamma} .
$$

Recall that for any $\eta>0$ we have that $\left|e^{i x}-1\right|<\max \left\{2,|x|^{\eta}\right\} \leq 2|x|^{\eta}$.

Recalling that $|f|_{(\alpha)}<\infty$, by taking $\eta=\varepsilon / \alpha$ we can bound

$$
\begin{aligned}
\Sigma_{f}^{(n), k} \leq & 2|t|^{\varepsilon / \alpha}|f|_{(\alpha)}^{\varepsilon / \alpha} \sum_{j_{1}, \ldots, j_{n}} \mu_{x}\left[j_{1}, \ldots, j_{n}\right] d\left(Z_{k}(x, \mathbf{j}), Z_{k}(y, \mathbf{j})\right)^{\varepsilon} d_{\lambda}\left(Z_{n}(x, \mathbf{j})\right)^{\gamma} \\
\leq & 2|t|^{\varepsilon / \alpha}|f|_{(\alpha)}^{\varepsilon / \alpha} \sum_{j_{1}, \ldots, j_{k}} \mu_{x}\left[j_{1}, \ldots, j_{k}\right] d\left(Z_{k}(x, \mathbf{j}), Z_{k}(y, \mathbf{j})\right)^{\varepsilon} d_{\lambda}\left(Z_{k}(x, \mathbf{j})\right)^{\gamma} \\
& \quad \times \sum_{j_{k+1}, \ldots, j_{n}} \mu_{Z_{k}(x, \mathbf{j})}\left[j_{k+1}, \ldots, j_{n}\right] \frac{d_{\lambda}\left(T_{j_{n}} \cdots T_{j_{k+1}}\left(Z_{k}(y, \mathbf{j})\right)\right)^{\gamma}}{d_{\lambda}\left(Z_{k}(y, \mathbf{j})\right)^{\gamma}} \\
\leq & 2|t|^{\varepsilon / \alpha}|f|_{(\alpha)}^{\varepsilon / \alpha} B_{n-k}(\gamma) \mathbb{E}_{x}\left(d\left(Z_{k}(x, \cdot), Z_{k}(y, \cdot)\right)^{\varepsilon} d_{\lambda}\left(Z_{k}(y, \mathbf{j})\right)^{\gamma}\right) \\
\leq & 2|t|^{\varepsilon / \alpha}|f|_{(\alpha)}^{\varepsilon / \alpha} B_{n-k}(\gamma) \mathbb{E}_{x}\left(\left\|T_{k}(\cdot)\right\|^{\varepsilon} \delta_{\lambda}\left(T_{k}(\cdot)\right)^{\gamma}\right) d(x, y)^{\varepsilon} d_{\lambda}(y)^{\gamma} .
\end{aligned}
$$


As $d_{\lambda}(y) \leq d_{\lambda}(x)$ and $\gamma<2 \beta$ it follows that

$$
\Sigma_{f}^{(n), k} \leq 2|t|^{\varepsilon / \alpha}|f|_{(\alpha)}^{\varepsilon / \alpha} B_{n-k}(\gamma) \mathbb{E}_{x}\left(\left\|T_{k}(\cdot)\right\|^{\varepsilon} \delta_{\lambda}\left(T_{k}(\cdot)\right)^{\gamma}\right) d(x, y)^{\varepsilon} d_{\lambda}(x)^{\beta} d_{\lambda}(y)^{\beta} .
$$

We can write

$$
\begin{aligned}
\Sigma_{\mu}^{(n)} & \leq \sum_{j_{1}, \ldots, j_{n}}\left|\mu_{x}\left[j_{1}, \ldots, j_{n}\right]-\mu_{y}\left[j_{1}, \ldots, j_{n}\right]\right|\left|w\left(Z_{n}(y, \mathbf{j})\right)\right| \\
& \leq|w|_{\gamma} \sum_{k=1}^{n} \Sigma_{\mu}^{(n), k}
\end{aligned}
$$

where

$$
\begin{aligned}
\Sigma_{\mu}^{(n), k}= & \sum_{j_{1}, \ldots, j_{n}} \mu_{Z_{k}(x, \mathbf{j})}\left[j_{k+1}, \ldots, j_{n}\right]\left|p_{j_{k}}\left(Z_{k-1}(x, \mathbf{j})\right)-p_{j_{k}}\left(Z_{k-1}(y, \mathbf{j})\right)\right| \\
& \times \mu_{y}\left[j_{1}, \ldots, j_{k-1}\right] d_{\lambda}\left(Z_{n}(y, \mathbf{j})\right)^{\gamma} \\
= & \sum_{j_{1}, \ldots, j_{k-1}}\left(\sum_{j_{k+1}, \ldots, j_{n}} \mu_{Z_{k}(x, \mathbf{j})}\left[j_{k+1}, \ldots, j_{n}\right] \frac{d_{\lambda}\left(T_{j_{n}} \cdots T_{j_{k+1}}\left(Z_{k}(x, \mathbf{j})\right)\right)^{\gamma}}{d_{\lambda}\left(Z_{k}(x, \mathbf{j})\right)^{\gamma}}\right) \\
& \quad \times \sum_{j_{k}} \frac{d_{\lambda}\left(T_{j_{k}}\left(Z_{k-1}(x, \mathbf{j})\right)^{\gamma}\right)}{d_{\lambda}\left(Z_{k-1}(x, \mathbf{j})\right)^{\gamma}} \frac{\left|p_{j_{k}}\left(Z_{k-1}(x, \mathbf{j})\right)-p_{j_{k}}\left(Z_{k-1}(y, \mathbf{j})\right)\right|}{d\left(Z_{k-1}(x, \mathbf{j}), Z_{k-1}(y, \mathbf{j})\right)^{\varepsilon}} \\
& \mu_{y}\left[j_{1}, \ldots, j_{k-1}\right] d\left(Z_{k-1}(x, \mathbf{j}), Z_{k-1}(y, \mathbf{j})\right)^{\varepsilon} d_{\lambda}\left(Z_{k-1}(x, \mathbf{j})\right)^{\gamma} \\
\leq & B_{n-k}(\gamma) A(\gamma, \varepsilon) \sum_{j_{1}, \ldots, j_{k-1}} \mu_{y}\left[j_{1}, \ldots, j_{k-1}\right] d\left(Z_{k-1}(x, \mathbf{j}), Z_{k-1}(y, \mathbf{j})\right)^{\varepsilon} d_{\lambda}\left(Z_{k-1}(x, \mathbf{j})\right)^{\gamma} \\
\leq & B_{n-k}(\gamma) A(\gamma, \varepsilon) \mathbb{E}_{y}\left(\left\|T_{k-1}(\cdot)\right\|^{\varepsilon} \delta_{\lambda}\left(T_{k-1}(\cdot)\right)^{\gamma}\right) d(x, y)^{\varepsilon} d_{\lambda}(y)^{\gamma} .
\end{aligned}
$$

As $d_{\lambda}(y) \leq d_{\lambda}(x)$ and $\gamma<2 \beta$ it follows that

$$
\Sigma_{\mu}^{(n), k} \leq B_{n-k}(\gamma) A(\gamma, \varepsilon) \mathbb{E}_{y}\left(\left\|T_{k-1}(\cdot)\right\|^{\varepsilon} \delta_{\lambda}\left(T_{k-1}(\cdot)\right)^{\gamma}\right) d(x, y)^{\varepsilon} d_{\lambda}(x)^{\beta} d_{\lambda}(y)^{\beta} .
$$

As $\varepsilon+\gamma<\varepsilon+2 \beta<1$ and by Lemma 4.2, the right-hand sides of (19), (20), (21) are finite. Hence there exists a constant $R_{n}>0$ such that

$$
\left|P_{t}^{n} w\right|_{\varepsilon, \beta} \leq \sup _{x} \mathbb{E}_{x}\left(\left\|T_{n}(\cdot)\right\|^{\varepsilon} \delta_{\lambda}\left(T_{n}(\cdot)\right)^{2 \beta}\right)|w|_{\varepsilon, \beta}+R_{n}|t|^{\varepsilon / \alpha}|w|_{\gamma} .
$$

Remark. By taking $n=1$ in Proposition 4.3 we see that $P_{t}$ maps $C_{\varepsilon, \beta, \gamma}$ into itself.

Consider the case $t=0$. Taking $n=n_{0}^{\prime}$ in Proposition 4.3 we have that

$$
\left|P_{t}^{n_{0}^{\prime}} w\right|_{\varepsilon, \beta} \leq r^{\prime}|w|_{\varepsilon, \beta}+R^{\prime}|t|^{\varepsilon / \alpha}|w|_{\gamma}
$$


for some $R^{\prime}>0$. It follows from Hennion's improvement $[\mathrm{H}]$ of the classical Ionescu-Marinescu-Tulcea theorem that $P^{n_{0}^{\prime}}$, and hence $P$, is a quasicompact operator. Hence we can decompose

$$
P=\sum_{\lambda \in G} \lambda \pi_{\lambda}+Q
$$

where $G$ is a finite group of eigenvalues of modulus $1, \pi_{\lambda}$ is the eigenprojection onto the corresponding eigenspace, $Q$ is the eigenprojection onto the remainder of the spectrum with $\rho(Q)<\rho$ for some $\rho \in(0,1)$, and $\pi_{\lambda} Q=Q \pi_{\lambda}=0$. The following result shows that, under (12), 1 is the only eigenvalue of modulus 1 for $P$.

\section{Lemma 4.4}

The only eigenvalue $\lambda$ of modulus 1 of $P: C_{\varepsilon, \beta, \gamma} \rightarrow C_{\varepsilon, \beta, \gamma}$ is $\lambda=1$ and the only eigenfunctions are constants.

Proof. This is proved in [Pe], albeit on a slightly different function space. For completeness we sketch the argument. Clearly $P 1=1$, so that 1 is an eigenvalue of $P$ and the constants are eigenfunctions. We decompose $P$ as in (23) and we show that $\pi_{1}(w)=\int w d \nu$ and $\pi_{\lambda}=0$ for $\lambda \neq 1$.

Suppose that $P w=w$, where $w \in C_{\varepsilon, \beta, \gamma}$ is bounded.

\section{Sub-lemma 4.5}

For each $x \in X$ we claim that there exists a set $\Sigma_{x} \subset \Sigma$ such that for all $\mathbf{j} \in \Sigma_{x}$ we have:

(i) $\lim _{n \rightarrow \infty} w\left(Z_{n}(x, \mathbf{j})\right)$ exists,

(ii) $\liminf _{n \rightarrow \infty} d\left(x_{0}, Z_{n}(x, \mathbf{j})\right)<\infty$,

(iii) for all $m \geq 1$ and all $i_{1}, \ldots, i_{m}$, we have that $w\left(Z_{n}(x, \mathbf{j})\right)=w\left(T_{i_{m}} \cdots T_{i_{1}} Z_{n}(x, \mathbf{j})\right)$ whenever $\mu_{Z_{n}(x, \mathbf{j})}\left[i_{1}, \ldots, i_{m}\right] \neq 0$.

Let $x, y \in X$. For each $\mathbf{j} \in \Sigma_{x}$, by (ii) we can choose a sequence $n_{k}=$ $n_{k}(\mathbf{j})$ such that $Z_{n_{k}}(x, \mathbf{j}) \rightarrow x_{\mathbf{j}}$ for some $x_{\mathbf{j}} \in X$. As $w$ is continuous, by (iii) we have that $w\left(x_{\mathbf{j}}\right)=w\left(T_{i_{m}} \cdots T_{i_{1}} x_{\mathbf{j}}\right)$ whenever $\mu_{x_{\mathbf{j}}}\left[i_{1}, \ldots, i_{m}\right] \neq$ 0 . Similarly, for each $\mathbf{j}^{\prime} \in \Sigma_{y}$, we can find $y_{\mathbf{j}^{\prime}} \in X$ such that $w\left(y_{\mathbf{j}^{\prime}}\right)=$ $w\left(T_{i_{m}} \cdots T_{i_{1}} y_{\mathbf{j}^{\prime}}\right)$ whenever $\mu_{y_{\mathbf{j}^{\prime}}}\left[i_{1}, \ldots, i_{m}\right] \neq 0$.

By $(12)$, choose $\mathbf{i}=\left(i_{1}, i_{2}, \ldots\right) \in \Sigma$ such that for all $m, \mu_{x_{\mathbf{j}}}\left[i_{1}, \ldots, i_{m}\right]$, $\mu_{y_{\mathbf{j}^{\prime}}}\left[i_{1}, \ldots, i_{m}\right] \neq 0$. Then, as the $p_{j}$ are continuous, (iii) is satisfied for sufficiently large $n_{k}$. Hence

$$
\begin{aligned}
& \left|w\left(x_{\mathbf{j}}\right)-w\left(y_{j}\right)\right| \\
& \quad=\left|w\left(T_{i_{m}} \cdots T_{i_{1}} x_{\mathbf{j}}\right)-w\left(T_{i_{m}} \cdots T_{i_{1}} y_{\mathbf{j}^{\prime}}\right)\right| \\
& \quad \leq|w|_{\varepsilon, \beta}\left\|T_{i_{m}} \cdots T_{i_{1}}\right\|^{\varepsilon} d_{\lambda}\left(T_{i_{m}} \cdots T_{i_{1}}\right)^{2 \beta} d\left(x_{\mathbf{j}}, y_{\mathbf{j}^{\prime}}\right)^{\varepsilon} d_{\lambda}\left(x_{\mathbf{j}}\right)^{\beta} d_{\lambda}\left(y_{\mathbf{j}^{\prime}}\right)^{\beta} \\
& \quad \leq C\left(x_{\mathbf{j}}, y_{\mathbf{j}^{\prime}}\right) \mathbb{E}_{x}\left(\left\|T_{m}(\mathbf{i})\right\|^{\varepsilon} \delta_{\lambda}\left(T_{m}(\mathbf{i})\right)^{2 \beta}\right) .
\end{aligned}
$$


Taking $m=q n_{0}^{\prime}$ where $n_{0}^{\prime}$ is given by (15) we see that this expression is bounded by $C\left(x_{\mathbf{j}}, y_{\mathbf{j}^{\prime}}\right)\left(r^{\prime}\right)^{q}$. Letting $q \rightarrow \infty$ shows that $w\left(x_{\mathbf{j}}\right)=w\left(y_{\mathbf{j}^{\prime}}\right)$ whenever $\mathbf{j} \in \Sigma_{x}$ and $\mathbf{j}^{\prime} \in \Sigma_{y}$. As $w\left(x_{\mathbf{j}}\right)=\lim _{n_{k}} w\left(Z_{n_{k}}(x, \mathbf{j})\right)$ and $w(x)=$ $\mathbb{E}_{x}\left(w\left(x_{x_{\mathbf{j}}}\right)\right)$, and similarly for $y$, it follows that $w(x)=w(y)$. Hence $w$ is constant.

Let $M_{n}=n^{-1} \sum_{k=0}^{n-1} P^{k}$. Then $\left\|M_{n}-\pi_{1}\right\| \rightarrow 0$ as $n \rightarrow \infty$ (here $\|\cdot\|$ denotes the operator norm on $\left.C_{\varepsilon, \beta, \gamma}\right)$. As $\left|M_{n} w\right|_{\infty} \leq|w|_{\infty}$, it follows that $\pi_{1} w$ is bounded. By the above, $\pi_{1} w$ is constant.

If $P w=w$ and $w \in C_{\varepsilon, \beta, \gamma}$ is not bounded, then define $w_{c}(x)=w(x)$ if $|w(x)| \leq c$ and $w_{c}(x)=c w(x) /|w(x)|$ if $|w(x)|>c$. Then $w_{c} \in C_{\varepsilon, \beta, \gamma}$ is bounded. Hence $\pi_{1} w_{c}$ is constant. Note that $\left|M_{n} w_{c}(x)-\pi_{1} w_{c}(x)\right| \leq$ $\left\|M_{n}-\pi_{1}\right\|\left\|w_{c}\right\|_{\varepsilon, \beta, \gamma} d_{\lambda}(x)^{\gamma}$. By dominated convergence, $M_{n} w_{c} \rightarrow M_{n} w$ as $c \rightarrow \infty$. Hence $\pi_{1} w_{c} \rightarrow \pi_{1} w$ as $c \rightarrow \infty$, so that $\pi_{1} w$ is constant.

It remains to prove Sublemma 4.5.

Proof of Sublemma 4.5. Let $\mathcal{B}_{n}$ denote the sub- $\sigma$-algebra of $\Sigma$ generated by cylinder sets of length $n$. Then it is straightforward to check from the definitions that if $P w=w$ then

$$
\mathbb{E}_{x}\left(w\left(Z_{n}(x, \mathbf{j})\right) \mid \mathcal{B}_{n}\right)=P\left(w\left(Z_{n}(x, \mathbf{j})\right)\right)=w\left(Z_{n}(x, \mathbf{j})\right) .
$$

Hence $w\left(Z_{n}(x, \cdot)\right)$ is a martingale with respect to $\mathcal{B}_{n}$. Property (i) then follows from standard properties of martingales.

Property (ii) is a simple consequence of the contraction on average assumption and (10).

Note that

$$
\begin{aligned}
\mathbb{E}_{x} & \left(\sum_{n=0}^{\infty} \sum_{i_{1}, \ldots, i_{m}} \mu_{Z_{n}(x, \cdot)}\left[i_{1}, \ldots, i_{m}\right]\left(w\left(T_{i_{m}} \cdots T_{i_{1}} Z_{n}(x, \cdot)\right)-w\left(Z_{n}(x, \cdot)\right)\right)^{2}\right) \\
& =\sum_{n=0}^{\infty} \mathbb{E}_{x}\left(\sum_{i_{1}, \ldots, i_{m}} \mu_{Z_{n}(x, \cdot)}\left[i_{1}, \ldots, i_{m}\right]\left(w\left(T_{i_{m}} \cdots T_{i_{1}} Z_{n}(x, \cdot)\right)-w\left(Z_{n}(x, \cdot)\right)\right)^{2}\right) \\
& =\sum_{n=0}^{\infty} \mathbb{E}_{x}\left(w\left(Z_{n+m}(x, \cdot)\right)^{2}\right)-\mathbb{E}_{x}\left(w\left(Z_{n}(x, \cdot)\right)^{2}\right) \\
& \leq 2 m|w|_{\infty}
\end{aligned}
$$

where we have used the fact that $\mathbb{E}_{x}\left(\left(w\left(Z_{n+m}(x, \cdot)\right)-w\left(Z_{n}(x, \cdot)\right)\right)^{2}\right)=$ $\mathbb{E}_{x}\left(w\left(Z_{n+m}(x, \cdot)\right)^{2}\right)-\mathbb{E}_{x}\left(w\left(Z_{n}(x, \cdot)\right)^{2}\right)$. Hence the first integrand in the expression above is finite $\mu_{x}$-a.e., hence the summand converges to zero $\mu_{x}$-a.e.

Now suppose that $P w=\lambda w$ where $\lambda \in G, w \in C_{\varepsilon, \beta, \gamma}$. Introduce a new operator $\hat{P}$ defined on $X \times \mathbb{N}$ by

$$
\hat{P} W(x, n)=\sum_{j} p_{j}(x) W\left(T_{j} x, n+1\right) .
$$


By taking $W(x, n)=\lambda^{-n} w(x)$, we see that $\hat{P} W=W$ if and only if $P w=$ $\lambda w$. A similar argument to that above then shows that $w$ is zero.

\section{$\S 4.2 \quad$ Perturbation of the spectrum of $P_{t}$}

We want to study how the spectrum of $P_{t}$ behaves for $t$ in a neighbourhood of 0 . To do this, we use the following perturbation result of Keller and Liverani:

Theorem 4.6 ([KL])

Suppose that $(B,\|\cdot\|)$ is a Banach space equipped with a second norm $|\cdot| \leq\|\cdot\|$ (we do not require $(B,|\cdot|)$ to be complete). Let $P_{t}$ be a oneparameter family of bounded linear operators. Suppose that

(i) the inclusion $\iota:(B,\|\cdot\|) \hookrightarrow(B,|\cdot|)$ is compact;

(ii) there exists an interval $J^{\prime}, 0 \in J^{\prime}$, and $n_{0}>0$ such that for $t \in J^{\prime}$, $P_{t}^{n_{0}}$ satisfies a uniform Lasota-Yorke condition: there exists $\rho \in(0,1)$ and $R>0$ such that for all $w \in B, t \in J$,

$$
\left\|P_{t}^{n_{0}} w\right\| \leq \rho\|w\|+R|w|
$$

(iii) $\left\|\left|P_{t}-P_{0} \|\right| \rightarrow 0\right.$ as $t \rightarrow 0$ (here, if $Q: B \rightarrow B$ is a bounded linear operator, then $\left.\left\|\left|Q\|\|=\sup _{w \in B}\right| Q w \mid /\right\| w \|\right)$.

Then there exists an interval $J \subset J^{\prime}$ containing 0 such that if $t \in J$ then $P_{t}$ is quasi-compact. Suppose in addition that $P_{0}$ has a unique simple maximal eigenvalue at 1 . Then $P_{t}$ has a unique simple maximal eigenvalue $\lambda_{t}$ with corresponding eigenprojection $\pi_{t}$. For $t \in J$, the dependence $t \mapsto \lambda_{t}$ is continuous, and $\left\|\left|\pi_{t}-\pi_{0}\right|\right\| \rightarrow 0$. Moreover, there exists $\rho_{0}<1$ such that if $Q_{t}$ denotes the projection onto the remainder of the spectrum of $P_{t}$, then $Q_{t}$ has spectral radius $\rho\left(Q_{t}\right) \leq \rho_{0}$, for all $t \in J$.

To apply Theorem 4.6, we need to study how $P_{t}-P$ varies in an appropriate norm. As is observed in a similar context in [HH2],

$$
\begin{aligned}
\left|P_{t} w(x)-w(x)\right| & \leq \sum_{j} p_{j}(x)\left|e^{i t f\left(T_{j} x\right)}-1\right|\left|w\left(T_{j} x\right)\right| \\
& \leq \sum_{j} p_{j}(x)|t|^{\varepsilon}\left|f\left(T_{j} x\right)\right|^{\varepsilon}|w|_{\gamma} \delta_{\lambda}\left(T_{j}\right)^{\gamma} d_{\lambda}(x)^{\gamma} .
\end{aligned}
$$

If $X$ is not compact, then $d_{\lambda}(x)$ is unbounded. Hence $t \mapsto P_{t}$ is not a priori continuous in the $|\cdot|_{\gamma}$-topology. For this reason we work with the norms $\|\cdot\|_{\varepsilon, \beta}^{(1)}$ and $|\cdot|^{(1)}$.

By Lemma 3.4, hypothesis (i) of Theorem 4.6 holds. 
For an operator $Q: C_{\varepsilon, \beta, \gamma} \rightarrow C_{\varepsilon, \beta, \gamma}$ we define

$$
\|Q\| \|=\sup \left\{\frac{|Q w|^{(1)}}{\|w\|_{\varepsilon, \beta}^{(1)}} \mid w \in C_{\varepsilon, \beta, \gamma}\right\} .
$$

\section{Lemma 4.7}

There exists a constant $C>0$ such that

$$
||\left|P_{t}-P\right||| \leq C|t|^{\varepsilon} .
$$

Proof. Note that if $w \in C_{\varepsilon, \beta, \gamma}$ then

$$
\begin{aligned}
\left|P_{t} w-P w\right| & \leq \sum_{j} p_{j}(x)\left|e^{i t f\left(T_{j} x\right)}-1\right|\left|w\left(T_{j} x\right)\right| \\
& \leq \sum_{j} p_{j}(x)|t|^{\varepsilon}\left|f\left(T_{j} x\right)\right|^{\varepsilon}|w|_{\gamma} \delta_{\lambda}\left(T_{j}\right)^{\gamma} d_{\lambda}(x)^{\gamma} .
\end{aligned}
$$

From (13) we have that

$$
\left|f\left(T_{j} x\right)\right|^{\varepsilon} \leq\left|f\left(x_{0}\right)\right|^{\varepsilon}+|f|_{(\alpha)}^{\varepsilon} d\left(T_{j} x, x_{0}\right)^{\varepsilon \alpha} \leq C \delta_{\lambda}\left(T_{j}\right)^{\varepsilon \alpha} d_{\lambda}(x)^{\varepsilon \alpha}
$$

for some constant $C>0$. Hence

$$
\nu\left(\left|P_{t} w-w\right|\right) \leq C|w|_{\gamma}|t|^{\varepsilon} \mathbb{E}_{x}\left(\delta\left(T_{j} x\right)^{\varepsilon \alpha+\gamma}\right) \int d_{\lambda}(x)^{\varepsilon \alpha+\gamma} d \nu .
$$

By definition, $|w|_{\gamma}<\|w\|_{\varepsilon, \beta, \gamma}$. By Lemma 3.3 and the fact that (14) implies that $\varepsilon \alpha+\gamma<1$ so that the two integrals in (24) are finite, it follows that $\nu\left(\left|P_{t} w-w\right|\right) \leq C^{\prime}|t|^{\varepsilon}\|w\|_{\varepsilon, \beta}^{(1)}$.

Hence hypothesis (ii) of Theorem 4.6 holds.

By Lemma 3.3 there exists $C>0$ such that $|\cdot|_{\gamma} \leq C\left(|\cdot|_{\varepsilon, \beta}+|\cdot|^{1}\right)$. From (22) we have that

$$
\left|P_{t}^{n_{0}} w\right|_{\varepsilon, \beta} \leq\left(r^{\prime}+C R^{\prime}|t|^{\varepsilon / \alpha}\right)|w|_{\varepsilon, \beta}+C R^{\prime}|t|^{\varepsilon / \alpha}|w|^{(1)} .
$$

Choose $\rho$ with $r^{\prime}<\rho<1$. Then there exists an interval $J^{\prime}$ containing 0 such that if $t \in J^{\prime}$ then $r^{\prime}+C R^{\prime}|t|^{\varepsilon / \alpha}<\rho$. Hence hypothesis (iii) of Theorem 4.6 holds.

By Lemma 4.4, $P$ has a simple maximal eigenvalue at 1 . Hence by Theorem 4.6 there exists an interval $J, 0 \in J$, and $\rho_{0}<1$ such that for $t \in J$ we can write

$$
P_{t}=\lambda_{t} \pi_{t}+Q_{t}
$$

where $t \mapsto \lambda_{t}$ is continuous, $\pi_{t}$ is a one-dimensional projection operator with $\lim _{t \rightarrow 0}\left\|\left|\pi_{t}-\nu\right|\right\|=0$, and $Q_{t}$ is the projection onto the remainder of the spectrum and has spectral radius at most $\rho_{0}$. As $\pi_{t} Q_{t}=Q_{t} \pi_{t}=0$, it follows that $P_{t}^{n}=\lambda_{t}^{n} \pi_{t}+Q_{t}^{n}$. 


\section{$\S 5$ Stable laws}

Stable distributions are generalisations of the Gaussian distribution. Given a sequence of normally distributed independent random variables $X_{i}$ it is well-known that the partial sums $S_{n}=X_{0}+\cdots+X_{n-1}$ have the property that $n^{-1 / 2} S_{n}$ is also normally distributed. The stable laws are characterised by this behaviour: a suitable rescaling of independent partial sums has the same distribution. More precisely, we have the following definition:

Definition. A distribution function $F$ is called stable if, for any $a_{1}, a_{2}>0$ and any $b_{1}, b_{2}$, there exist constants $a>0$ and $b$ such that $F\left(a_{1} x+b_{1}\right) *$ $F\left(a_{2} x+b_{2}\right)=F(a x+b)$, where $*$ denotes convolution.

It is known that four parameters completely determine a stable law [IL]: $Y_{p, \beta, b, c}$ denotes the stable law with order $p \in(0,2)$, symmetry $\beta \in[-1,1]$, origin $b \in \mathbb{R}$ and scaling factor $c>0$. We often identify stable distributions up to translation and scaling, denoting them simply by $Y_{p, \beta}$.

The tail behaviour of a stable law is encoded in its order $p$. For $p \in$ $(0,1) \cup(0,2)$, there are constants $c_{1}, c_{2} \geq 0$ (not both zero) such that $P\left(Y_{p, \beta}>t\right)=\left(c_{1}+o(1)\right) t^{-p}$ and $P\left(Y_{p, \beta}<t\right)=\left(c_{2}+o(1)\right) t^{-p}$.

Recall that if a random variable has distribution $F$ then the characteristic function $\phi$ of this distribution is given by

$$
\phi(t)=\int_{-\infty}^{\infty} e^{i t x} d F(x) .
$$

In general, an explicit formula for the density of a stable distribution is only known in a handful of special cases. However, explicit formulæ for their characteristic functions are known.

Theorem 5.1 ([IL])

A distribution $Y$ is stable of order $p \in(0,1) \cup(1,2)$ if and only if its characteristic function $\phi_{Y}(t)$ can be written in the form

$$
\phi_{Y}(t)=\exp \left(i b t-c|t|^{p}\left(1-i \beta \operatorname{sign}(t) \tan \left(\frac{\pi}{2} p\right)\right)\right),
$$

where $\beta, b$ and $c$ are constants.

There is a corresponding formula for stable laws of order 1 [AD1].

We will also need the notion of slowly varying function.

Definition. A function $l: \mathbb{R} \rightarrow \mathbb{R}$ is said to be slowly varying if for every $t \in \mathbb{R}$

$$
\lim _{x \rightarrow \infty} \frac{l(t x)}{l(x)}=1
$$


Definition. We say that a function $f$ (with distribution $F$ ) is in the domain of attraction of a stable law of order $p \in(0,2)$ if there is a slowly varying function $l: \mathbb{R} \rightarrow \mathbb{R}$ and constants $c_{1}, c_{2} \geq 0$, not both zero, such that

$$
1-F(t)=\frac{1}{t^{p}}\left(c_{1}+o(1)\right) l(t), F(-t)=\frac{1}{t^{p}}\left(c_{2}+o(1)\right) l(t)
$$

as $t \rightarrow \infty$.

The expansion of the characteristic function of a function in the domain attraction of a stable law was studied by Ibragimov and Linnik for $p \neq 1$ [IL] and by Aaronson and Denker [AD1] for $p=1$ in dimension one and for all $p$ for multidimensional distributions. For simplicity, in what follows we will focus on real-valued functions and the case $p \in(0,1) \cup(0,2)$.

In $\S 6$ we relate the characteristic function of an observation $f$ defined on the IFS to the expansion of the maximal eigenvalue of $P_{t}$. We shall use the following criterion.

\section{Theorem 5.2 ([IL])}

Let $F$ be a distribution with characteristic function $\phi_{F}(t)$. Suppose that $p \neq 1$ and let $Y=Y_{p, \beta, b, c}$ be the stable law with characteristic function given by (25). Then a necessary and sufficient condition for $F$ to be in the domain of attraction of the stable law $Y$ is that in the neighbourhood of the origin

$$
\phi_{F}(t)=\exp \left(i b t-c|t|^{p} l(t)\left(1-i \beta \operatorname{sign}(t) \tan \left(\frac{\pi}{2} p\right)\right)\right),
$$

where $l(t)$ is slowly varying as $t \rightarrow 0$.

\section{$\S 6$ Expansion of the maximal eigenvalue}

When $\sigma^{2}(f)<\infty$ then one has the following expansion of the maximal eigenvalue $\lambda_{t}$ of $P_{t}$ :

$$
\lambda_{t}=\nu(f) t+\frac{\sigma^{2}(f)}{2} t^{2}+o\left(t^{2}\right)
$$

from which the Central Limit Theorem then follows. Under additional hypotheses, the $o\left(t^{2}\right)$ term can be improved and Berry-Esseen bounds can be proved. This is discussed in [HH2] in the case of random walks on semigroups of Lipschitz mappings that contract on average, with a place-independent probability.

When $\sigma^{2}(f)$ is infinite we have the following asymptotic expansion of $\lambda_{t}$.

\section{Theorem 6.1 (Expansion of the maximal eigenvalue)}

For $p \in(0,1) \cup(1.2)$ suppose that $f$ has distribution $F$ and is in the domain of attraction of a stable law $Y=Y_{p, \beta, b, c}$ of order $p$. Let $\lambda_{t}$ be the maximal 
eigenvalue of the perturbed operator $P_{t}$. Then there is a slowly varying function $l$ such that

$$
\operatorname{Re} \log \lambda_{t}=-\operatorname{sign}(t)|t|^{p} c l\left(t^{-1}\right)+o\left(|t|^{p} l\left(t^{-1}\right)\right)
$$

and

$$
\operatorname{Im} \log \lambda_{t}=t b-\operatorname{sign}(t)|t|^{p} c \beta \tan \left(\frac{p \pi}{2}\right)+o\left(t^{p} l\left(t^{-1}\right)\right),
$$

where $\beta=\left(c_{2}-c_{1}\right) /\left(c_{1}+c_{2}\right)$ with $c_{1}, c_{2}$ as in $(26), b$ is given by

$$
b= \begin{cases}0 & \text { for } p \in(0,1), \\ \int_{-\infty}^{\infty} x d F(x) & \text { for } p \in(1,2)\end{cases}
$$

and $c=\left(c_{1}+c_{2}\right) \pi / 2$.

Proof (sketch). The proof follows closely ideas in [AD2] (which in turn makes use of ideas in $[\mathrm{N}]$ ). We indicate the modifications required.

Since $f$, with distribution $F$, is in the domain of attraction of a stable law $Y_{p, \beta}$ there is a slowly varying function $l: \mathbb{R} \rightarrow \mathbb{R}$ and constants $c_{1}, c_{2} \geq 0$, not both zero, such that (26) holds.

The proof consists of estimating $1-\lambda_{t}$, where $\lambda_{t}$ is the maximal eigenvalue of $P_{t}$, and then using the fact that $\log \left(\lambda_{t}\right)=\log \left(1+\left(\lambda_{t}-1\right)\right)=$ $\left(\lambda_{t}-1\right)+O\left(\left|\lambda_{t}-1\right|^{2}\right)$.

Let $w_{t}$ denote the maximal eigenfunction of $P_{t}$ corresponding to the eigenvalue $\lambda_{t}$ so that $P_{t} w_{t}=\lambda_{t} w_{t}$. We normalise $w_{t}$ so that $\int w_{t} d \nu=1$. Let $\mathcal{F}$ denote $f^{-1}(\mathcal{B})$ where $\mathcal{B}$ denotes the $\sigma$-algebra of Borel subsets of $\mathbb{R}$. We define $\tilde{w}_{t}$ by $\tilde{w}_{t} \circ f=\mathbb{E}\left(w_{t} \mid \mathcal{F}\right)$. Note that

$$
\begin{aligned}
\lambda_{t} & =\lambda_{t} \nu\left(w_{t}\right)=\nu\left(\lambda_{t} w_{t}\right)=\nu\left(P_{t} w_{t}\right)=\nu\left(P\left(e^{i t f} w_{t}\right)\right) \\
& =\nu\left(e^{i t f} w_{t}\right)=\nu\left(e^{i t f} \mathbb{E}\left(w_{t} \mid \mathcal{F}\right)\right)=\int e^{i t x} \tilde{w}_{t} d F(x) .
\end{aligned}
$$

Similarly,

$$
1=\int \tilde{w}_{t} d F(x)
$$

so that $d F_{t}=\tilde{w}_{t} d F$ is a probability measure on $\mathbb{R}$. Hence

$$
1-\lambda_{t}=\int_{-\infty}^{\infty}\left(1-e^{i t x}\right) d F_{t}(x) .
$$

The proof now proceeds as in $[\mathrm{AD} 2, \mathrm{~N}]$. 


\section{$\S 7$ Distributional stable limit theorems}

We are now in position to prove our main result.

\section{Theorem 7.1}

For $p \in(0,1) \cup(1,2)$, suppose that $f$ is continuous, $|f|_{(\alpha)}<\infty$ and is in the domain of attraction of a stable law $Y$ of order $p$, satisfying (26). Then for any $x \in X$,

$$
\mu_{x}\left\{\mathbf{j} \in \Sigma \mid \frac{S_{n} f(x, \mathbf{j})-a_{n}}{b_{n}}<t\right\} \rightarrow \int_{-\infty}^{t} d Y,
$$

as $n \rightarrow \infty$ with $a_{n}=0$ if $p<1$ and $a_{n}=n \nu(f)$ if $p>1$, and $b_{n}^{p}=n l\left(b_{n}\right)$.

Proof. By replacing $f$ by $f-\nu(f)$ there is no loss in generality in assuming that $a_{n}=0$.

It is well-known that a sequence of random variables converges in distribution if and only if their corresponding characteristic functions converge pointwise at continuity points. The characteristic function of $Y=Y_{p, \beta}$ is

$$
\mathbb{E}\left(e^{i t Y}\right)=\exp \left(-c|t|^{p}\left(1-i \beta \operatorname{sign}(t) \tan \left(\frac{p \pi}{2}\right)\right)\right) .
$$

Hence it is sufficient to prove that

$$
\mathbb{E}_{x}\left(\exp \left(i t \frac{S_{n} f(x, \cdot)}{b_{n}}\right)\right) \rightarrow \exp \left(-c|t|^{p}\left(1-i \beta \operatorname{sign}(t) \tan \left(\frac{p \pi}{2}\right)\right)\right)
$$

as $n \rightarrow \infty$.

By $\S 4.2$, we can write $P_{s}^{n}=\lambda_{s}^{n} \pi_{s}+Q_{s}^{n}$ for $s \in J$, a neighbourhood of 0. Fix $t$. Then $t / b_{n} \in J$ for sufficiently large $n$. Recalling (17), we note that

$$
\begin{aligned}
& \left|P_{\frac{t}{b_{n}}}^{n} 1(x)-\exp \left(-c|t|^{p}\left(1-i \beta \operatorname{sign}(t) \tan \left(\frac{p \pi}{2}\right)\right)\right)\right| \\
& =\left|\lambda_{\frac{t}{b_{n}}}^{n} \pi_{\frac{t}{b_{n}}}(1)(x)+Q_{\frac{t}{b_{n}}}^{n}(1)(x)-\exp \left(-c|t|^{p}\left(1-i \beta \operatorname{sign}(t) \tan \left(\frac{p \pi}{2}\right)\right)\right)\right| \\
& \leq\left|\lambda_{\frac{t}{b_{n}}}^{n} \pi_{\frac{t}{b_{n}}}(1)(x)-\exp \left(-c|t|^{p}\left(1-i \beta \operatorname{sign}(t) \tan \left(\frac{p \pi}{2}\right)\right)\right)\right|+\left|Q_{\frac{t}{b_{n}}}^{n}(1)(x)\right| \\
& \leq\left|\lambda_{\frac{t}{b_{n}}}^{n}-\exp \left(-c|t|^{p}\left(1-i \beta \operatorname{sign}(t) \tan \left(\frac{p \pi}{2}\right)\right)\right)\right| \\
& \quad+\left|\lambda_{\frac{t}{b_{n}}}^{n}\right| \cdot\left|\pi_{\frac{t}{b_{n}}}(1)(x)-1\right|+\left|Q_{\frac{t}{b_{n}}}^{n}(1)(x)\right| .
\end{aligned}
$$

As $\left|\lambda_{t / b_{n}}^{n}\right|$ is bounded above by 1 and $\left\|\left|\pi_{t}-\nu\right|\right\| \rightarrow 0$, we have

$$
\left|\lambda_{\frac{t}{b_{n}}}^{n}\right| \cdot\left|\pi_{\frac{t}{b_{n}}}(1)(x)-1\right| \rightarrow 0
$$

as $n \rightarrow \infty$. Moreover,

$$
\left|Q_{\frac{t}{b_{n}}}^{n}(1)(x)\right| \rightarrow 0
$$


as $n \rightarrow \infty$ as $\left\|Q_{\frac{t}{b_{n}}}^{n}\right\| \leq \rho_{0}^{n}$ provided $n$ is sufficiently large. It remains to show that the first term in (27) converges to 0 .

It follows from Theorem 6.1 that

$$
\operatorname{Re} \log \lambda_{\frac{t}{b_{n}}}=-n \operatorname{sign}(t) c\left|\frac{t}{b_{n}}\right|^{p} l\left(\frac{b_{n}}{t}\right)+o\left(\left|\frac{t}{b_{n}}\right|^{p} l\left(\frac{b_{n}}{t}\right)\right) .
$$

and

$$
\operatorname{Im} \log \lambda_{\frac{t}{b_{n}}}=-n \operatorname{sign}(t) c\left|\frac{t}{b_{n}}\right|^{p}\left(\beta l\left(\frac{b_{n}}{t}\right) \tan \left(\frac{p \pi}{2}\right)\right)+o\left(\left|\frac{t}{b_{n}}\right|^{p} l\left(\frac{b_{n}}{t}\right)\right) .
$$

Note that

$$
o\left(\left|\frac{t}{b_{n}}\right|^{p} l\left(\frac{b_{n}}{t}\right)\right)=o\left(\frac{t^{p}}{n} \frac{l\left(b_{n} / t\right)}{l\left(b_{n}\right)}\right) .
$$

As $l$ is slowly varying, $l\left(b_{n} / t\right) / l\left(b_{n}\right) \rightarrow 1$ as $n \rightarrow \infty$. Hence (28) converges to 0 as $n \rightarrow \infty$.

Similarly,

$$
-n \operatorname{sign}(t) c\left|\frac{t}{b_{n}}\right|^{p} l\left(\frac{b_{n}}{t}\right)=-\operatorname{sign}(t) c|t|^{p} \frac{l\left(b_{n} / t\right)}{l\left(b_{n}\right)} \rightarrow-\operatorname{sign}(t) c|t|^{p}
$$

and

$$
\begin{aligned}
-n \operatorname{sign}(t) c\left|\frac{t}{b_{n}}\right|^{p} \beta l\left(\frac{b_{n}}{t}\right) \tan \left(\frac{p \pi}{2}\right) & =-\operatorname{sign}(t) c|t|^{p} \beta \frac{l\left(b_{n} / t\right)}{l\left(b_{n}\right)} \tan \left(\frac{p \pi}{2}\right) \\
& \rightarrow-\operatorname{sign}(t) c|t|^{p} \beta \tan \left(\frac{p \pi}{2}\right)
\end{aligned}
$$

as $n \rightarrow \infty$. This completes the proof.

\section{$\S 8$ Local stable limit theorems}

\section{§8.1 Arithmeticity and cohomology of observations}

The local distributional limiting behaviour of the sequence of observations $f\left(Z_{n}(x, \cdot)\right)$ depends on whether $f$ essentially takes values in a lattice.

Definition. A function $f: X \rightarrow \mathbb{R}$ is said to be arithmetic if there exists $t_{0} \neq 0$ such that $P_{t_{0}}$ has an eigenvalue of modulus 1 , i.e. there exists $r \in$ $[0,2 \pi), w \in C_{\varepsilon, \beta, \gamma}, w \neq 0$, such that $P_{t_{0}} w=e^{i r} w$. Otherwise, we say that $f$ is non-arithmetic.

We have the following characterisation of arithmeticity. We say that two functions $f_{1}, f_{2}$ are cohomologous if there exists a function $h$ such that $f_{1} T_{j}=f_{2} T_{j}+h-h T_{j}$ for all $j$.

\section{Proposition 8.1}

Suppose that $f$ is continuous and that $\int f d \nu=0$. Then the following are equivalent: 
(i) $f$ is arithmetic.

(ii) $f$ is cohomologous to a function taking values in a lattice.

Proof. If $f$ is cohomologous to a lattice-valued function then there exists $t_{0} \neq 0$ such that $t_{0} f T_{j}=r+k T_{j}=h-h T_{j}$ where $r \in \mathbb{R}$ and $k$ is $2 \pi \mathbb{Z}$-valued. It follows that $P_{t_{0}}\left(e^{i h}\right)=e^{i r} e^{i h}$ so that $f$ is arithmetic.

The converse is a standard convexity argument. Suppose that for some $t_{0} \neq 0$ there exists a non-zero $w \in C_{\varepsilon, \beta, \gamma}$ such that $P_{t_{0}} w=e^{i r} w$. Then $|w| \leq P(|w|)$. As $\nu(|w|) \leq \nu(P(|w|))=\nu(|w|)$ it follows that $|w|$ is a constant, which we may take to be $1, \nu$-a.e. Writing $w=e^{i h}$ we have that $P_{t_{0}}\left(e^{i h}\right)=\sum_{j} p_{j}(x) e^{i t_{0} f\left(T_{j} x\right)+i h\left(T_{j} x\right)}=e^{i r} e^{i h}$, a convex sum of complex numbers of modulus 1 . Hence this sum is trivial, i.e. $f T_{j}=r+k T_{j}+h-h T_{j}$ for some lattice valued function $k$.

\section{$\S 8.2 \quad$ Local stable limit theorems}

In this section we prove the local stable limit theorem in the case where $f$ is in the domain of attraction of a stable law of order $p \in(0,1) \cup(1,2)$.

\section{Theorem 8.2}

Let $f$ be a continuous non-arithmetic function with $|f|_{(\alpha)}<\infty$ and $\nu(f)=0$. Suppose that $f$ satisfies (26) and is in the domain of attraction of a stable law $Y_{p}$ with density $y_{p}$. Fix $x \in X$. Then for any $a, b \in \mathbb{R}, a<b$ we have

$$
\lim _{n \rightarrow \infty}\left|b_{n} \mu_{x}\left\{\mathbf{j} \in \Sigma \mid S_{n} f(x, \mathbf{j}) \in z+[a, b]\right\}-y_{p}\left(z / b_{n}\right)(b-a)\right|=0
$$

uniformly in $z \in \mathbb{R}$, where $b_{n}^{p}=b l\left(b_{n}\right)$.

Proof. Define the sequence of measures $m_{n}$ on $\mathbb{R}$ by defining how they integrate continuous functions: if $w: \mathbb{R} \rightarrow \mathbb{R}$ is continuous

$$
\int w d m_{n}=\mathbb{E}_{x}\left(\frac{b_{n}}{y_{p}\left(z / b_{n}\right)} \int w\left(-z+S_{n} f(x, \cdot)\right)\right)
$$

for a continuous function $w: \mathbb{R} \rightarrow \mathbb{R}$. To prove the theorem it is sufficient to prove that $m_{n}$ weak* converges to Lebesgue measure on $\mathbb{R}$. Let $H$ denote the space of continuous functions $w: \mathbb{R} \rightarrow \mathbb{R}$ such that the Fourier transform $\hat{w}$ is compactly supported. To prove that $m_{n}$ weak* converges to Lebesgue measure, it is sufficient to prove that $m_{n}(w) \rightarrow \int w(t) d t$ for all $w \in H[\mathrm{Br}]$. Define

$$
A_{n}(z)=b_{n} \mathbb{E}_{x}\left(w\left(-z+S_{n} f(x, \cdot)\right)\right)-y_{p}\left(z / b_{n}\right) \int_{-\infty}^{\infty} w(t) d t .
$$

Thus it is sufficient to prove that $\left|A_{n}(z)\right| \rightarrow 0$ as $n \rightarrow \infty$ uniformly in $z \in \mathbb{R}$. 
We denote the Fourier transform of $w$ by

$$
\hat{w}(s)=\int_{-\infty}^{\infty} w(t) e^{-i s t} d t .
$$

Then by the inversion formula we have

$$
w(t)=\frac{1}{2 \pi} \int_{-\infty}^{\infty} \hat{w}(s) e^{i s t} d s .
$$

Moreover, by Theorem 5.1 we have that

$$
y_{p}(z)=\frac{1}{2 \pi} \int_{-\infty}^{\infty} \phi_{p}(t) e^{-i t z} d t
$$

where

$$
\phi_{p}(t)=\exp \left(-c|t|^{p}(1-i \beta \operatorname{sign}(t) \tan (p \pi / 2))\right) .
$$

Suppose that $\hat{w}$ is supported on $[-\delta, \delta]$. Then for any $\alpha \in(0, \delta)$ we can write

$$
\begin{aligned}
2 \pi & A_{n}(z) \\
= & b_{n} \mathbb{E}_{x}\left(\int_{-\infty}^{\infty} \hat{w}(t) e^{i t\left(-z+S_{n} f(x, \cdot)\right)} d t\right)-\hat{w}(0) \int_{-\infty}^{\infty} \phi_{p}(t) e^{-\frac{i t z}{b_{n}}} d t \\
= & b_{n} \int_{-\delta}^{\delta} \hat{w}(t) \int_{\Sigma} e^{i t S_{n} f(x, \cdot)} d \mu_{x} e^{-i t z} d t-\hat{w}(0) \int_{-\infty}^{\infty} \phi_{p}(t) e^{-\frac{i t z}{b_{n}}} d t \\
= & \int_{-\delta b_{n}}^{\delta b_{n}} \hat{w}\left(\frac{t}{b_{n}}\right) P_{\frac{t}{b_{n}}}^{n} 1(x) e^{-\frac{i t z}{b_{n}}} d t-\hat{w}(0) \int_{-\infty}^{\infty} \phi_{p}(t) e^{-\frac{i t z}{b_{n}}} d t \\
= & \int_{|t| \leq \alpha b_{n}}\left(\hat{w}\left(\frac{t}{b_{n}}\right) P_{\frac{t}{b_{n}}}^{n} 1(x)-\hat{w}(0) \phi_{p}(t)\right) e^{-\frac{i t z}{b_{n}}} d t \\
& \quad+\int_{\alpha b_{n} \leq|t| \leq \delta b_{n}} \hat{w}\left(\frac{t}{b_{n}}\right) P_{\frac{t}{b_{n}}}^{n} 1(x) e^{-\frac{i t z}{b_{n}}} d t \\
& \quad+\int_{|t| \geq \alpha b_{n}} \hat{w}(0) \phi_{p}(t) e^{-\frac{i t z}{b_{n}}} d t .
\end{aligned}
$$

Recalling that $P_{t}^{n}=\lambda_{t}^{n} \pi_{t}+Q_{t}^{n}$ we see that

$$
\begin{aligned}
(29)= & \int_{|t| \leq \alpha b_{n}}\left(\hat{w}\left(\frac{t}{b_{n}}\right) \lambda_{\frac{t}{b_{n}}}^{n} \pi_{\frac{t}{b_{n}}} 1(x)-\hat{w}(0) \phi_{p}(t)\right) e^{-\frac{i t z}{b_{n}}} d t \\
& +\int_{|t| \leq \alpha b_{n}} \hat{w}\left(\frac{t}{b_{n}}\right) Q_{\frac{t}{b_{n}}}^{n} 1(x) e^{-\frac{i t z}{b_{n}}} d t .
\end{aligned}
$$

By $\S 4.2$ we can choose $\alpha \in(0, \delta)$ sufficiently small so that there exists $\rho<1$ and a constant $C>0$ such that $\left\|Q_{t / b_{n}}^{n}\right\| \leq C \rho_{0}^{n}$ and $\left|\lambda_{t / b_{n}}\right| \in(\rho, 1]$ for all $|t| \leq \alpha b_{n}$. Notice that from the proof of Theorem 7.1 that $\lambda_{t / b_{n}}^{n} \rightarrow \phi_{p}(t)$ as $n \rightarrow \infty$. It follows from $\S 4.2$ that $\pi_{t / b_{n}} 1 \rightarrow \nu(1)=1$ as $n \rightarrow \infty$. By continuity, $\hat{w}\left(t / b_{n}\right) \rightarrow \hat{w}(0)$ as $n \rightarrow \infty$. Hence the integrand in (32) converges 
to 0 as $n \rightarrow \infty$. As, moreover, the integrand is bounded in modulus by an integrable function, it follows from the Dominated Convergence Theorem that (32) converges to 0 as $n \rightarrow \infty$.

Noting that as $b_{n}^{p}=n l\left(b_{n}\right)$ for a slowly varying function $l$, it follows that $b_{n}=O\left(n^{\frac{1}{p}+\epsilon}\right)$ for any $\epsilon>0$. Hence

$$
|(33)| \leq C \rho_{0}^{n} b_{n} \rightarrow 0
$$

as $n \rightarrow \infty$.

Similarly, we can estimate (30). For each $t \in[\alpha, \delta], P_{t}$ does not have 1 as an eigenvalue. Hence there exists $\eta<1$ and a constant $C>0$ such that $\left\|P_{t}^{n}\right\| \leq C \eta^{n}$. Hence

$$
|(30)| \leq C \eta^{n} b_{n} \rightarrow 0
$$

as $n \rightarrow \infty$.

Clearly (31) converges to 0 as $n \rightarrow \infty$ as $b_{n} \rightarrow \infty$ and $\phi_{p} \in L^{1}$.

Noting that none of the constants above depend on $z$ we see that $A_{n}(z) \rightarrow$ 0 as $n \rightarrow \infty$, uniformly in $z \in \mathbb{R}$.

Remark. The proof of the local central limit theorem is similar, cf. [BPD], with $e^{-t^{2} / 2}$ replacing $y_{p}(t)$.

Remark. One can also formulate and prove a stable local limit theorem in the case of an arithmetic Hölder function $f$; cf. [AD2].

\section{$\S 9$ Random difference equations}

Let $a_{j} \in \mathbb{R}, a_{j}>0, b_{j} \in \mathbb{R}$. random difference equation is determined by the equation

$$
z_{j+1}=a_{j+1} z_{j}+b_{j+1}
$$

where the $\left(a_{j}, b_{j}\right)$ are i.i.d. pairs of real numbers. There is a great deal of literature on the solution to such equations and their applications ([Ke, DF], for example). In the case where the $\left(a_{j}, b_{j}\right)$ are chosen independently from a finite or countable set then (34) can be viewed as an IFS by taking $T_{j}(x)=$ $a_{j} x+b$, chosen with probability $p_{j}$.

The affine IFS $\left(T_{j}, p_{j}\right)$ satisfies $(1)$ precisely when $\sum p_{j} a_{j}<1$. (The technical hypothesis $(10)$ holds provided $\sum p_{j} b_{j}<\infty$.) In this case, the tail behaviour of the invariant measure $\nu$ is well-known.

Theorem 9.1 ([Ke, Gol] )

Suppose the affine IFS $\left(T_{j}, p_{j}\right)$ contracts on average and that the closed subgroup of $\mathbb{R}$ generated by $\log a_{j}$ is $\mathbb{R}$. Define $\theta_{0}$ by $\sum p_{j} a_{j}^{\theta_{0}}=1$. Suppose that for some $\epsilon>0$ we have $\sum p_{j} a_{j}^{\theta_{0}+\epsilon}, \sum p_{j} b_{j}^{\theta_{0}+\epsilon}<\infty$. Then $\nu([t, \infty)) \sim \frac{1}{t^{\theta_{0}}}$ as $t \rightarrow \infty$. 
Taking $f(x)=x^{\alpha}$ where $\alpha \in(0,1]$ we see that $f$ satisfies (7) with $p=$ $\theta_{0} / \alpha$, and so the distributional and local limit Theorems (when $f$ is nonarithmetic) above hold in this case.

More generally, the case of a random walk on the affine group of $\mathbb{R}$ has been studied [GP]. In this case the probability used to choose the $T_{j}$ need not be supported on a discrete set of maps. In the case where $f(x)=x$, a complete analysis of the expansion of the maximal eigenvalue of $P_{t}$, and an precise identification of the stable limit law can be achieved [GP].

\section{References}

[AD1] J. Aaronson and M. Denker, Characteristic functions of random variables attracted to 1-stable laws, Ann. Prob., 26 (1998), 399-415.

[AD2] J. Aaronson and M. Denker, Local limit theorems for partial sums of stationary sequences generated by Gibbs-Markov maps, Stoch. Dyn., 1 (2001), 193-237.

[BDEG] M.F. Barnsley, S.G. Demko, J.H. Elton and J.S. Geronimo, Invariant measures for Markov processes arising from iterated function systems with place-dependent probabilities. Ann. Inst. H. Poincaré Probab. Statist., 24 (1988), 367-394.

[Br] L. Breiman, Probability, Addison-Wesley, Reading, Mass., 1968.

[BPD] A. Broise, M. Peigné and F. Dal'bo, Études spectrales d'opérateurs de transfert et applications, Astérisque 238 (1996), Soc. Math. de France.

[DF] P. Diaconis and D. Freedman, Iterated random functions, SIAM Rev., 41 (1999), 45-76.

[E1] J.H. Elton, An ergodic theorem for iterated maps, Ergod. Th. \& Dynam. Sys., 7 (1987), 481-488.

[E2] J.H. Elton, A multiplicative ergodic theorem for Lipschitz maps, Stoch. Proc. Appl., 34 (1990), 39-47.

[Gol] C.M. Goldie, Implicit renewal theory and tails of solutions of random equations, Ann. Appl. Prob., 1 (1991), 126-166.

[Gou] S. Gouëzel, Central limit theorem and stable laws for intermittent maps, Prob. Th. Rel. Fields, 128 (2004), 82-122.

[GP] Y. Guivarc'h and E. le Page, On spectral properties of a family of transfer operators and convergence to stable laws for affine random walks, Ergod. Th. \& Dynam. Sys., 28 (2008), 423-446.

$[\mathrm{H}] \quad$ H. Hennion, Décomposition spectrale des opérateurs de Doeblin-Fortet, Proc. Amer. Math. Soc., 69 (1993), 627-634.

[HH1] H. Hennion and L. Hervé, Limit theorems for Markov chains and stochastic properties of dynamical systems by quasi-compactness, Springer Lecture Notes in Mathematics, 1766 (2001).

[HH2] H. Hennion and L. Hervé, Central limit theorems for iterated random Lipschitz mappings, Ann. Prob., 32 (2004), 1934-1984.

[IL] I. Ibragimov and Yu. Linnik, Independent and Stationary Sequences of Random Variables, Wolters-Noordhodd Publishing, Groningen, 1971. 
[KL] G. Keller and C. Liverani, Stability of the spectrum for transfer operators, Ann. Sc. Norm. Super. Pisa, 28 (1999), 141-152.

[Ke] H. Kesten, Random difference equations and renewal theory for products of random matrices, Acta Math, 131 (1973), 207-248.

[N] S.V. Nagaev, Some limit theorems for stationary Markov chains, Theor. Probab. Appl. 2 (1957), 378-406.

[Pe] M. Peigné, Iterated function systems and spectral decomposition of the associated Markov operator, Publ. Inst. Rech. Math. Rennes 1993, Univ. Rennes I, Rennes, 1993.

[W] C.P. Walkden, Invariance principles for iterated maps that contract on average, Trans. Amer. Math. Soc., 359 (2007), 1081-1097.

Sara Santos, School of Mathematics, The University of Manchester, Oxford Road, Manchester M13 9PL, U.K., email: sara.i.santos@gmail.com

Charles Walkden, School of Mathematics, The University of Manchester, Oxford Road, Manchester M13 9PL, U.K., email: charles.walkden@manchester.ac.uk 